\title{
Linking Topography to Tonotopy in the Mouse Auditory Thalamocortical Circuit
}

\author{
Troy A. Hackett, ${ }^{1 \star}$ Tania Rinaldi Barkat, ${ }^{2 \star}$ Barbara M. J. 0’Brien, ${ }^{1}$ Takao K. Hensch, ${ }^{2}$ and Daniel B. Polley ${ }^{3}$ \\ ${ }^{1}$ Vanderbilt Kennedy Center for Research on Human Development, Department of Hearing and Speech Sciences, Vanderbilt University School of Medicine, \\ Nashville, Tennessee 37232, ${ }^{2}$ Center for Brain Science, Department Molecular and Cellular Biology, Harvard University, Cambridge, Massachusetts 02138, \\ and ${ }^{3}$ Eaton-Peabody Laboratory, Massachusetts Eye and Ear Infirmary, Department of Otology and Laryngology, Harvard Medical School, Boston MA
} 02114

The mouse sensory neocortex is reported to lack several hallmark features of topographic organization such as ocular dominance and orientation columns in primary visual cortex or fine-scale tonotopy in primary auditory cortex (AI). Here, we re-examined the question of auditory functional topography by aligning ultra-dense receptive field maps from the auditory cortex and thalamus of the mouse in vivo with the neural circuitry contained in the auditory thalamocortical slice in vitro. We observed precisely organized tonotopic maps of best frequency $(\mathrm{BF})$ in the middle layers of $\mathrm{AI}$ and the anterior auditory field as well as in the ventral and medial divisions of the medial geniculate body (MGBv and MGBm, respectively). Tracer injections into distinct zones of the BF map in AI retrogradely labeled topographically organized MGBv projections and weaker, mixed projections from MGBm. Stimulating MGBv along the tonotopic axis in the slice produced an orderly shift of voltage-sensitive dye (VSD) signals along the AI tonotopic axis, demonstrating topography in the mouse thalamocortical circuit that is preserved in the slice. However, compared with BF maps of neuronal spiking activity, the topographic order of subthreshold VSD maps was reduced in layer IV and even further degraded in layer II/III. Therefore, the precision of AI topography varies according to the source and layer of the mapping signal. Our findings further bridge the gap between in vivo and in vitro approaches for the detailed cellular study of auditory thalamocortical circuit organization and plasticity in the genetically tractable mouse model.

\section{Introduction}

The auditory cortex is functionally organized to contain spatial representations of basic stimulus attributes and stimulusevoked response properties (for review, see Schreiner and Winer, 2007). Some of these spatially ordered representations, such as tonotopy, can be distributed over hundreds of thousands of neurons, while other features, such as the representations for pitch, sound level, spectral integration, and binaural or temporal modulation preference are clustered into a patchwork of spatially discrete modules grouped by laminae or cortical subregion (Middlebrooks et al., 1980; Schreiner and Mendelson, 1990; Polley et al., 2007; Atencio and Schreiner, 2010a; Bendor and Wang, 2010). The synaptic, neurochemical and cell-autonomous properties that distinguish one cortical module from the next have remained mysterious because this cellular level of analysis is most effectively performed in vitro. Yet, studies of specialized sensory modules require, by defini-

\footnotetext{
Received Oct. 11, 2010; revised Nov. 29, 2010; accepted Dec. 27, 2010.

This work was supported by National Institutes of Health Grants DC009836 (D.B.P.) and DC04318 (T.A.H.); Core Research for Evolutional Science and Technology, Japan Science \& Technology Agency (T.K.H.); the Human Frontiers Science Program (T.K.H.); and the Harvard Society of Fellows (T.R.B.). We thank Michelle Young and Dr. Vivek Khatri for technical assistance and fruitful discussion.

*T.A.H. and T.R.B. contributed equally to this work.

Correspondence should be addressed to Dr. Daniel B. Polley, Eaton-Peabody Laboratory, Massachusetts Eye and Ear Infirmary, 243 Charles Street, Boston, MA 02114. E-mail: Daniel_polley@meei.harvard.edu.

DOI:10.1523/JNEUROSCI.5333-10.2011

Copyright $\odot 2011$ the authors $\quad 0270-6474 / 11 / 312983-13 \$ 15.00 / 0$
}

tion, an intact connection between the neuron and the sensory receptor epithelium.

Although in vivo whole-cell recordings have begun to uncover the interplay between synaptic excitation and inhibition that shape cortical receptive fields (Wehr and Zador, 2003; Wu et al., 2006; Montgomery and Wehr, 2010; Zhou et al., 2010), a detailed understanding of thalamocortical microcircuits, on the order of what has been achieved in the cochlear nucleus, would require a regional and cell type-specific analysis performed in vitro as well as in vivo (Godfrey et al., 1975; Young and Brownell, 1976; Manis, 1990; Reyes et al., 1994; Oertel et al., 2000). The mouse auditory thalamocortical slice offers a promising approach to understand the cellular bases for regional variations in the representational capacities of auditory cortex neurons. In this acute preparation, the connection between the ventral medial geniculate body (MGBv) and auditory cortex (AI) is preserved, permitting an in vitro analysis of thalamocortical synaptic transmission that can be mechanistically dissected with the genetic toolbox uniquely available in the mouse (Cruikshank et al., 2002, 2010; de la Rocha et al., 2008; Llano and Sherman, 2009). One of the principal aims of this study was to provide a Rosetta Stone for translating the spatial geometry of a sensory feature map delineated in AI and MGBv of intact mice onto the same neural circuitry present in the thalamocortical slice.

Tonotopy, the spatially ordered gradient of preferred sound frequency, is a reasonable first choice to be explored both in vivo and in vitro. Tonotopy is a highly conserved organizational feature documented in AI and MGBv of at least 20 mammalian 
species over the past 4 decades (for review, see Kaas, 2011). In the mouse auditory cortex, however, the existence of clearly organized tonotopic maps is controversial, with two studies reporting orderly gradients spanning AI and anterior auditory field (AAF) (Stiebler et al., 1997; Zhang et al., 2005) and two recent studies describing a heterogeneous organization of best frequency (BF) in the same fields (Bandyopadhyay et al., 2010; Rothschild et al., 2010). Moreover, the presence or absence of a tonotopic organization in $\mathrm{MGBv}$ of the mouse has never been reported.

The goals of the present study were to determine whether a precisely organized tonotopic map exists in MGBv and core fields of the auditory cortex, how the basic pattern of thalamocortical afferents give rise to such tonotopic order (or lack thereof) in the cortex, and finally whether an organizational pattern established in vivo could also be demonstrated in the thalamocortical brain slice. We were surprised to find that spatially organized activity patterns in the slice motivated additional experiments in the intact preparation, which may shed some light on the debate surrounding the existence of precise tonotopy in the mouse auditory cortex.

\section{Materials and Methods}

\section{In vivo neurophysiology studies}

Neurophysiological data collection. All procedures were approved by Vanderbilt University and Harvard University animal care and use committees and followed the guidelines established by the National Institutes of Health for the care and use of laboratory animals. Female C57BL/6 mice aged 4-7 weeks were brought to a surgical plane of anesthesia using a combination of pentobarbital sodium $(50 \mathrm{mg} / \mathrm{kg}$ followed by $10-15$ $\mathrm{mg} / \mathrm{kg}$ supplements as needed) and chlorprothixene $(0.2 \mathrm{mg})$. Multiunit responses were recorded from the middle cortical layers of AI ( $420-440$ $\mu \mathrm{m}$ from pial surface) with epoxylite-coated tungsten microelectrodes (2.0 M $\Omega$ at $1 \mathrm{kHz}$; FHC) and from MGB with 16-channel silicon probes $\left(177 \mu \mathrm{m}^{2}\right.$ contact area, $50 \mu \mathrm{m}$ intercontact separation; Neuronexus Technologies). Frequency response areas (FRAs) were measured with pseudo-randomly presented tone pips of variable frequency (5.5-45.3 $\mathrm{kHz}$ in 0.1 octave increments, $20 \mathrm{~ms}$ duration, $5 \mathrm{~ms}$ raised cosine onset/ offset ramps, $600 \mathrm{~ms}$ intertrial interval) and level $[0-60 \mathrm{~dB}$ sound pressure level (SPL) in $5 \mathrm{~dB}$ increments] delivered from a free field electrostatic speaker placed $12 \mathrm{~cm}$ from the contralateral ear.

Auditory core fields (AI and AAF) were identified by an unmistakable caudal-to-rostral mirror reversal in tonotopy bounded by sites with poor or abruptly shifted frequency tuning. The rostral-to-caudal tonotopic zone is restricted to a narrow swath of cortical tissue with a precise layout that cannot be reliably predicted by coordinates from bregma or vascular landmarks. For MGB recordings, the silicon probe was inserted through the auditory cortex at $15^{\circ}$ off the horizontal plane under stereotaxic guidance to match the plane of the section used in tracer reconstruction and thalamocortical slice experiments. To avoid recording from the dorsal division of the MGB, the probe was initially inserted lateral to the auditory core fields, $\sim 3.5 \mathrm{~mm}$ caudal to bregma. The ventral edge of the MGB was identified by documenting the most lateral cortical insertion point that yielded driven responses from the MGB, some $2.5-3.0 \mathrm{~mm}$ from the cortical surface. Reconstruction of lesions and electrode tracks confirmed that this corresponded to the ventral shell of the MGB. To target the MGBv and the medial MGB (MGBm), the silicon probe was inserted $0.5 \mathrm{~mm}$ medial to this point, a trajectory that reliably corresponded to the center of the MGBv, as evidenced by histologic reconstruction of lesions and electrode tracks.

FRAs were reconstructed across the full rostral-to-caudal extent of the MGB by making successive penetrations rostral and caudal to the starting position (50-100 $\mu \mathrm{m}$ between penetrations) until the recording probe had advanced beyond the caudal and rostral poles of the MGB. FRAs were also compared along the full lateral-to-medial extent of the MGBv and MGBm by documenting variations in response properties across the linear array of contact sites spanning $0.75 \mathrm{~mm}$. In some cases, electrolytic lesions were made at various rostral-caudal positions in the MGB identified with the silicon probe. FRAs were measured at different insertion depths with a tungsten microelectrode, and small lesions were made by passing $0.8 \mu \mathrm{A}$ of current for $12 \mathrm{~s}$ at one or two points of interest along the lateral-to-medial penetration (e.g., the lateral or medial extremes of tone-driven recording sites or reversals in frequency tuning).

Analysis of individual FRAs. We performed two traditional measurements of preferred frequency tuning: $\mathrm{BF}$, the tone frequency that elicited the greatest number of spikes across all sound levels, and characteristic frequency, the frequency to which the neuron was responsive at threshold. BF values were distributed across a broader range of frequencies, produced more orderly maps, and were completely objective in their definition. BF was therefore judged to be the better choice for preferred tuning in the present study. Onset latency was defined as the first $1 \mathrm{~ms}$ poststimulus onset time bin that populated a high-intensity region of the FRA near the BF ( $>40 \mathrm{~dB}$ SPL, \pm 0.5 octaves). The low- and highfrequency borders of the FRA were then determined at each tone level, and the bandwidth of the tuning was expressed relative to the minimum response threshold.

Reconstruction of tonotopic maps in auditory cortex and thalamus. Mapping the auditory cortex and thalamus was achieved by carefully marking the position of the recording electrode on a high-resolution photograph of the surface vasculature. For cortical maps, the topographic position was defined by fitting a line that connected the low-frequency BF region in AI and AAF, and calculating the position of each recording site along the line (see Fig. 1C). Topographic variation in BF was well fit with a quadratic function, with the peak of the fit line used to identify the boundary between AI and AAF. For MGB maps, caudal-to-rostral position was expressed relative to the caudal- and rostral-most penetrations that yielded a measurable FRA from two or more contiguous contacts on the silicon probe. In nearly all MGB penetrations, BFs increased, reached a plateau, and then decreased along the lateral-to-medial extent. The most medial recording site in the plateau portion of the function was defined as the BF reversal point. BF values lateral and medial to the reversal point were independently fit with linear functions, and the slope and range of the fit lines were calculated (see Fig. 5A).

Analysis of spatially distributed tone representations. All AI and AAF recording sites for a given mouse were divided into 18 equally spaced regions of interest (ROIs) along the rostral-to-caudal extent of each field. Similarly, positions of all MGBv recording sites in a given mouse were assigned to one of nine spatial zones spanning the caudolateral-torostromedial tonotopic gradient. Next, for each recording site, we determined whether a single tone drawn from a set of evenly spaced frequencies $(5.7,8.6,13.0,19.7,27.9$, or $36.8 \mathrm{kHz})$ and levels $(30,40,50$, or $60 \mathrm{~dB}$ SPL) was contained within the FRA. In so doing, we were able to determine the probability that a given spatial zone of AI, AAF, or MGBv contained a given tone/level combination in their receptive fields. Response probability functions were derived from all recording sites within a given brain structure (see Fig. $7 B-G$ ). For the purpose of direct comparison between spatial maps generated with voltagesensitive dye (VSD), the peak and half-maximum boundaries of the normalized response probability functions were calculated for each map individually (see Fig. $7 \mathrm{H}, I$ ).

\section{Retrograde tracer and lesion studies}

Dual retrograde tracer injection. For these experiments, 4-7-week-old C57BL/6 mice were anesthetized with ketamine $(90 \mathrm{mg} / \mathrm{kg}$ with $30 \mathrm{mg} / \mathrm{kg}$ supplements as needed) and medetomidine $(0.5 \mathrm{mg} / \mathrm{kg})$. Following the initial microelectrode mapping, retrograde tracers, cholera toxin $\beta$ subunit (CTB) conjugated to either Alexa 488 (green, catalog \#34775, Invitrogen) or 594 (red, catalog \#34777, Invitrogen) were injected into low and high domains of the BF map, respectively $(n=3)$. Glass pipettes with a $20-30 \mu \mathrm{m}$ tip diameter containing CTB tracer $(1 \%$ in $0.1 \mathrm{~m}$ phosphate buffer) and back-filled with mineral oil were attached to a Hamilton syringe. Small quantities (0.1-0.2 $\mu \mathrm{l}$ ) of each tracer were pressure injected into the middle layers of each BF domain, which subsequently spread to all layers. Upon completion of the injections, the surgical area was closed and the animal allowed to recover for $\sim 80 \mathrm{~h}$ before transcardial perfusion. 

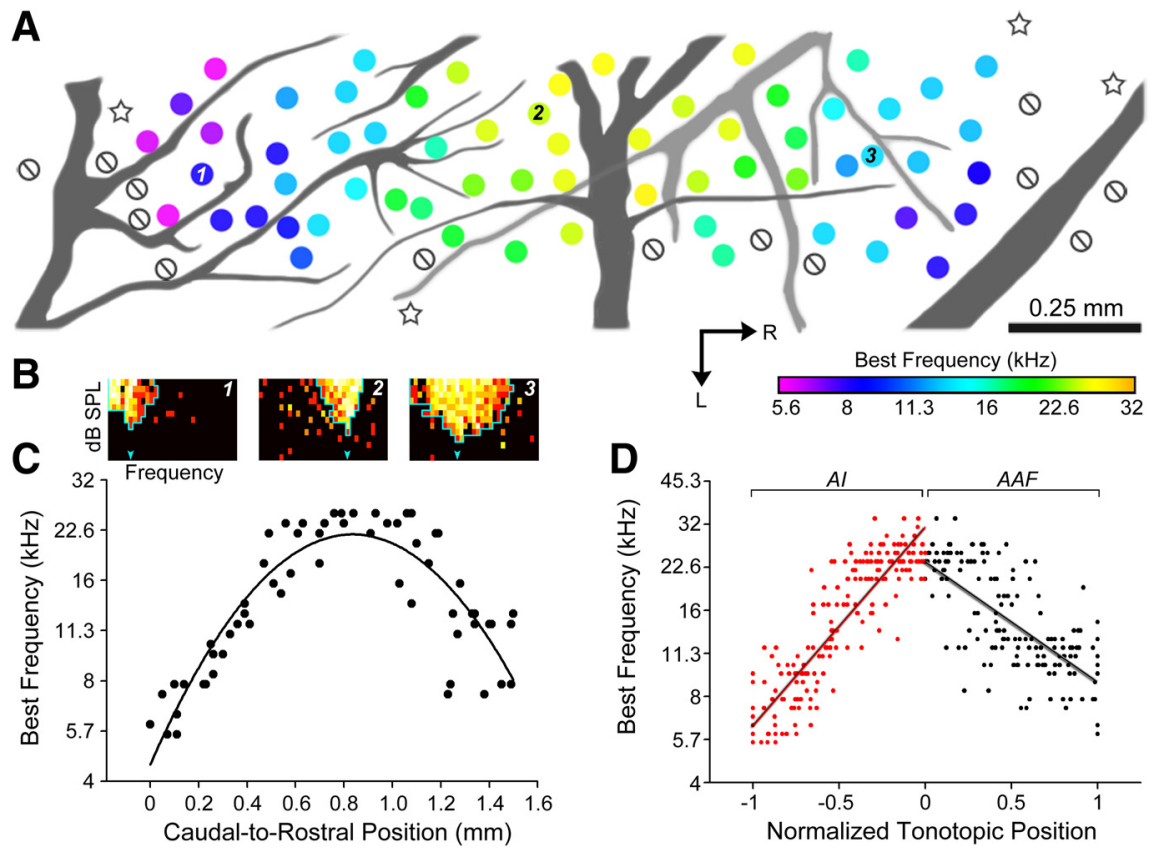

Figure 1. Tonotopic organization in the middle layers of Al and AAF. A, Positions of recording sites from the right hemisphere of a representative mouse are referenced to the branching patterns of the rhinal vein (dark gray) and middle cerebral artery (light gray). The color of each circle indicates the BF measured at that site, null symbol indicates no tuning, and stars indicate poorly tuned sites judged to be outside of Al or AAF. $\boldsymbol{B}$, Example FRAs measured at the numbered positions in $\boldsymbol{A}$. The downward arrow represents $B F$, and the outline represents the low- and high-frequency borders of the FRA at each SPL.C, BF measurements from the recording sites shown in $\boldsymbol{A}$ are plotted according to their position along a caudal-to-rostral line beginning at the caudal edge of Al. $\boldsymbol{D}$, Scatterplot of all BF values across normalized tonotopic position in Al and AAF. Linear fit lines are superimposed. R, Rostral; $L$, lateral. Scale bar, $0.25 \mathrm{~mm}$.

Histology. Animals were perfused through the heart with $\sim 100 \mathrm{ml}$ of each of the following solutions prepared in $0.1 \mathrm{~m}$ phosphate buffer, $\mathrm{pH}$ 7.4: $0.9 \%$ saline, cold $\left(4^{\circ} \mathrm{C}\right) 4 \%$ paraformaldehyde, and cold $\left(4^{\circ} \mathrm{C}\right) 4 \%$ paraformaldehyde plus $10 \%$ sucrose. Following perfusion, the brains were removed and placed in 30\% sucrose for $1-3 \mathrm{~d}$. Blocks containing auditory cortex were cut frozen at $40 \mu \mathrm{m}$ on a sliding microtome in the coronal plane (lesion cases) or in a modified horizontal plane (slice cases) that included Al and MGB, as described by Cruikshank et al. (2002). Alternating series of sections were processed for Nissl using thionin, cytochrome oxidase (lesion cases), immunohistochemistry for parvalbumin (slice cases), and fluorescent tracers (slice cases) (Hackett and de la Mothe, 2009).

The relative position of retrogradely labeled cell bodies was quantified independently in MGBv and MGBm from each section by fitting a rectangle with each boundary set by the locations of the most caudal, rostral, lateral, and medial cell bodies. The Cartesian coordinates for the red- and green-labeled cells were then expressed relative to these normalized boundaries. For lesion studies, we calculated the minimum distance separating the center of each lesion from the medial boundary separating the MGBv and MGBm.

In vitro voltage-sensitive dye studies

Acute auditory thalamocortical slice preparation. C57BL/6J mice (postnatal day 16-20) were rapidly decapitated without anesthesia and placed in ice-cold artificial CSF (ACSF). Slices (600 $\mu$ m thick) were sectioned on a vibratome using previously established methods (Cruikshank et al., 2002). Slices incubated at $35^{\circ} \mathrm{C}$ for at least 15 min were then maintained at room temperature $\left(20-22^{\circ} \mathrm{C}\right)$. The ACSF contained the following (in m): $125 \mathrm{NaCl}, 25$ glucose, $25 \mathrm{NaHCO}_{3}, 2.5 \mathrm{KCl}, 2 \mathrm{CaCl}_{2}, 1.25$ $\mathrm{NaH}_{2} \mathrm{PO}_{4}$, and $1 \mathrm{MgCl}_{2}$.

VSD imaging and analysis. Voltage-sensitive dye Di-4-ANEPPS (catalog \#D-1199, Invitrogen) dissolved at $0.01 \mathrm{mg} / \mathrm{ml}$ in dimethylsulfoxide stock solution was diluted $(0.6 \mu \mathrm{l} / \mathrm{ml})$ in ACSF, producing a final solution of $5 \mu \mathrm{g} / \mathrm{l}$ Di-4-ANEPPS. Slices were incubated (in $30 \mathrm{ml}$ ) before transfer to an ACSF (room temperature) chamber on an Olympus
MVX10 microscope with $1 \times 0.25$ numerical aperture objective. Excitation light from a shuttered $150 \mathrm{~W}$ halogen lamp (MHF-G150LR, Moritex) was bandpass filtered $(515 / 35 \mathrm{~nm})$ and reflected toward the sample by a $570 \mathrm{~nm}$ dichroic mirror. Emitted fluorescence was long-pass filtered $(590 \mathrm{~nm})$ and imaged using a MiCam Ultima CMOS-based camera (BrainVision and SciMedia).

Fluorescent signals were integrated across different ROIs through spatial averaging within a $125 \times 125 \mu \mathrm{m}$ area. Fluorescence change was normalized to resting fluorescence $\left(\Delta \mathrm{F} / \mathrm{F}_{0}\right)$. Discrete sites in the MGBv were activated with an ACSF-filled patch pipette (1 ms long, $0.5 \mathrm{~mA}$ square pulse) at $100 \mathrm{~ms}$ postrecording onset. Response amplitude was defined as maximal fluorescence change $(\Delta \mathrm{F} / \mathrm{F})$ per trial at a given ROI and then averaged across 10 trials. Variations between daily preparations were minimized by dividing responses by the maximum change in fluorescence measured in each set of experiments.

\section{Results}

Tonotopic organization in core fields of the auditory cortex

The spatial organization of BF was assessed across the caudal-to-rostral extent of AI and AAF with ultra-high-density mapping from 341 multiunit recording sites in 11 mice $(N=188$ sites in 6 mice and 153 sites in 5 mice for right and left hemisphere, respectively). Recordings targeted the middle cortical layers $(420-440 \mu \mathrm{m}$ from the pial surface). We observed a clear tonotopic organization for BF with low-frequency BFs of $\sim 6 \mathrm{kHz}$ forming the caudal boundary of $\mathrm{AI}$, a high-frequency mirror reversal at $\sim 32 \mathrm{kHz}$ forming the boundary between $\mathrm{AI}$ and $\mathrm{AAF}$, and a progressive shift to lower frequency BFs at the rostral border of AAF (Fig. $1 A$ ). Note that mice can hear and vocalize up to $100 \mathrm{kHz}$, yet the highest frequency cortical BF recorded in our entire sample was $34.3 \mathrm{kHz}$. Our recordings were performed at 4-7 weeks of age, several months before the onset of high-frequency hearing loss in C57BL/6 mice (Willott, 1986). In fact, the paucity of BFs $>40$ $\mathrm{kHz}$ has been routinely reported in the inferior colliculus and auditory cortex of several inbred and outbred mouse strains, and it is thought that these acoustic frequencies may be represented in specialized fields (Stiebler et al., 1997) or, intriguingly, according to their subharmonic cochlear distortion products (Portfors et al., 2009).

Representative FRAs from AI and AAF recording sites show well demarcated $\mathrm{V}$-shaped tuning profiles (Fig. $1 B$ ). BF changes were plotted across the caudal-to-rostral expanse of AI and AAF with an average sampling density of 40 recordings per millimeter, or one recording site every $25 \mu \mathrm{m}$. We observed an orderly parabolic shift in BF that was well fit by a quadratic function, with the peak used to define the high-frequency boundary separating AI and AAF (Fig. 1C). We then combined normalized maps from individual mice to represent 0 as the AI-AAF reversal point, -1 as the caudal low-frequency boundary of $\mathrm{AI}$, and +1 as the rostral low-frequency boundary of AAF (Fig. $1 D$ ). Using this approach, we found a significant increase in $\mathrm{BF}$ across the caudal-to-rostral extent of AI (one-way ANOVA, $F=34.9, p<1.10^{-6}$ ) and a 


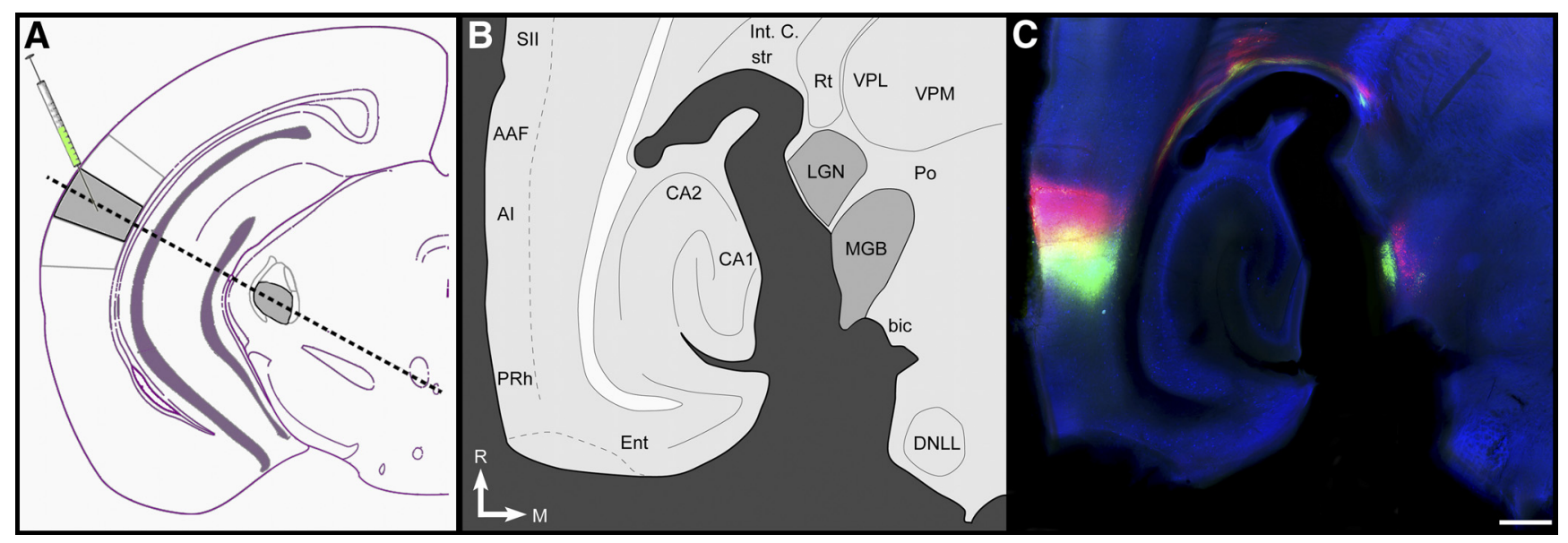

Figure 2. Tonotopic organization of MGBv projections to Al. $A$, Schematized coronal section through the mouse brain indicating the relative locations of Al and MGBv (gray shading), the position of (TB injections, and the plane of the section used to achieve the thalamocortical slice (dashed black line). $\boldsymbol{B}$, Schematic representation of major brain nuclei contained within the auditory thalamocortical brain slice. C, Auditory thalamocortical slice immunoreacted for parvalbumin (blue). CTB-green and CTB-red were injected into 7 and $22.6 \mathrm{kHz}$ domains in Al, respectively. SII, Secondary somatosensory cortex; Int C, internal capsule; str, superior thalamic radiation; Rt, reticular nucleus; VPL, ventral posterolateral thalamic nucleus; VPM, ventral posteromedial thalamic nucleus; LGN, lateral geniculate nucleus; $\mathrm{Po}$, posterior thalamic complex; bic, brachium of the inferior colliculus; DNLL, dorsal nucleus of the lateral lemniscus; Ent, entorhinal cortex; PRh, perirhinal cortex; R, rostral; M, medial. Scale bar, $0.25 \mathrm{~mm}$.

significant decrease in $\mathrm{BF}$ along the caudal-to-rostral extent of $\operatorname{AAF}(F=6.88, p<0.005)$.

\section{Tonotopic organization of MGBv projections to AI}

As a next step, we sought to determine whether tonotopy in AI arose from topographically organized MGB projections. To address this possibility, fluorescent retrograde tracers (CTB-red and CTB-green) were injected into a low- and a high-frequency region of AI that had been identified through microelectrode mapping, as described above (Fig. $2 \mathrm{~A}$ ). Following a waiting period of $\sim 80 \mathrm{~h}$ for retrograde transport, the brain was sectioned along the same plane used in auditory thalamocortical slice experiments (Cruikshank et al., 2002), which permitted visualization of $\mathrm{MGBv}$, the thalamocortical axon bundle, and AI (Fig. $2 B$ ). In the example shown in Figure 2C, CTB-green was injected into a 7.0 $\mathrm{kHz} \mathrm{BF}$ region and CTB-red was injected into a $22.6 \mathrm{kHz} \mathrm{BF}$ region. Qualitatively, one can appreciate that well segregated AI injection sites were innervated by compartmentalized axon bundles that originated from spatially separable populations of MGB neurons.

The projection patterns were examined in greater detail from this case (Fig. $3 A, B$ ) as well as a second case with injections into the low $(5.7 \mathrm{kHz})$ and high $(32.0 \mathrm{kHz}) \mathrm{BF}$ extremes of the AI map (Fig. $3 C, D$ ). In both cases, we observed dense, spatially separable projections from $\mathrm{MGBv}$ to each $\mathrm{BF}$ region in $\mathrm{AI}$ accompanied by a sparser, comingled projection from MGBm (Fig. $3 A, C$ ). Cell body locations were plotted against the anatomical boundaries of MGBv and MGBm in multiple sections across the dorsal-toventral extent of MGBv. A consistent pattern emerged from all sections in both cases: in MGBv, low-frequency projection neurons were located caudally and laterally, and high-frequency projection neurons were rostral and medial; MGBm neurons projecting to AI were not spatially separable (Fig. $3 B, D$ ).

Further quantification revealed that $75 \%$ of retrogradely labeled cell bodies were from MGBv, 24\% from MGBm, and the remaining $1 \%$ from the posterior thalamic complex. Lowfrequency projection neurons in $\mathrm{MGBv}$ were significantly more lateral (unpaired $t$ test, $p<1 \times 10^{-6}$ ) and caudal $\left(p<1 \times 10^{-6}\right.$ ) than high-frequency projection neurons (Fig. $3 E$ ). No significant difference in medial-lateral position was observed among low- and high-frequency projection neurons in MGBm ( $p=0.12)$, although high-frequency projection neurons were observed to be significantly more rostral $(p<0.005)$ (Fig. $3 F)$. Together, these data demonstrate that cortical projections from $\mathrm{MGBv}$ are topographically ordered and further suggest that MGBv may be tonotopically organized along a low-to-high, caudolateralto-rostromedial axis (Fig. 3G).

\section{Neurophysiological dissociation of MGBv and MGBm}

Findings from the tracer studies suggest three explicit predictions regarding tonotopic map orientation in the MGBv, as follows: (1) recordings from rostral-to-caudal positions along the lateral wall of the MGBv should yield a progressive downward shift in BF; (2) recordings across the lateral-to-medial extent of the MGBv should yield a progressive upward shift in BF; and (3) there should be no clear tonotopic organization within the MGBm. To test these predictions, we performed high-density multiunit mapping across the lateral-to-medial extent of the MGBv and MGBm using a multichannel silicon probe $(50 \mu \mathrm{m}$ separation between contacts). Penetrations were made throughout the rostral-to-caudal extent of the MGB (50-100 $\mu$ m between penetrations) at the same angle as the plane of section in the thalamocortical slice (Fig. 4A). Example FRAs shown in Figure 4A illustrate the drop in $\mathrm{BF}$ along the lateral wall of the MGBv between rostral, intermediate, and caudal recording locations (left column). Furthermore, regardless of the BF at the most lateral recording position, $\mathrm{BF}$ increased across the first few medial recording locations. However, it was equally clear that BFs subsequently decreased as the recording location moved to deeper, more medial areas of the MGB.

Did this high-frequency mirror reversal in $\mathrm{BF}$ indicate that recordings had crossed into the MGBm, or did it reveal a $\mathrm{BF}$ organization that was not suggested from the tracer studies? To address this question, we identified positions along the rostralto-caudal extent of the MGB that yielded clear mirror reversals with the silicon probe (Fig. $4 B$, gray arrow) and then inserted a conventional tungsten microelectrode along the same trajectory (Fig. $4 \mathrm{~B}$, white arrow) to make small electrolytic lesions lateral and/or medial to the BF reversal. An example of one such experiment is shown in Figure 4, $B$ and $C$, where a lateral lesion was 

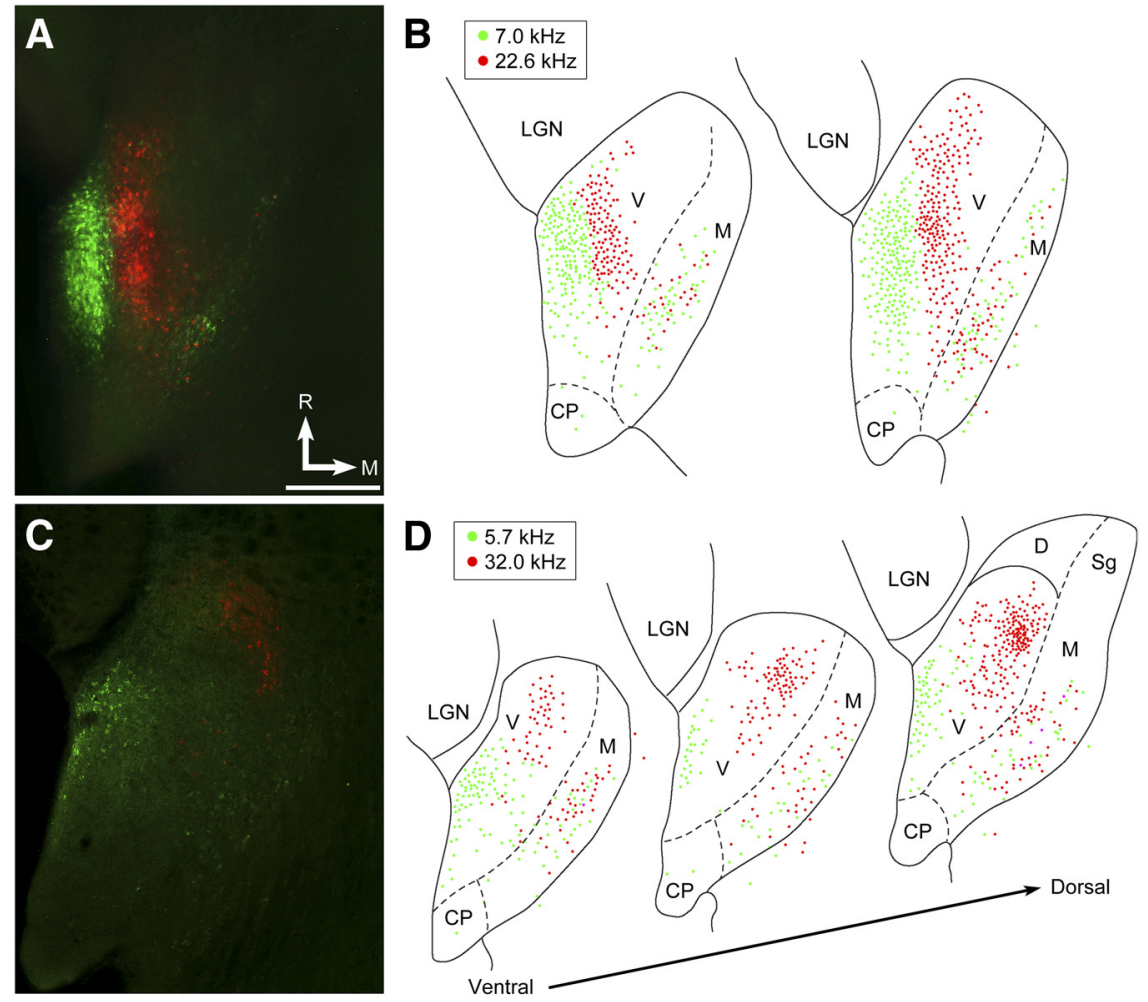

E

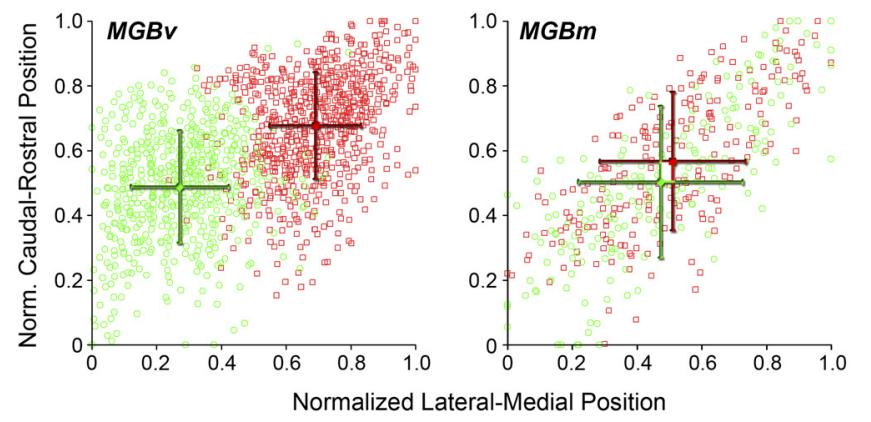

G

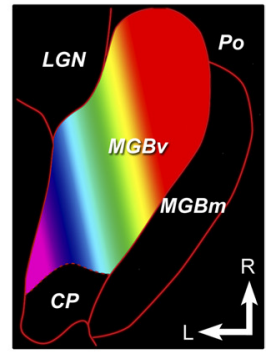

Figure 3. Topographic projections from MGBv, but not MGBm, to $A l$. $\boldsymbol{A}-\boldsymbol{D}$, Fluorescence microscopy $(\boldsymbol{A}, \boldsymbol{C})$ and retrogradely labeled cell body reconstructions $(\boldsymbol{B}, \boldsymbol{D})$ from a mouse with CTB injected into 7 and $22.6 \mathrm{kHz}$ Al domains $(\boldsymbol{A}, \boldsymbol{B})$ or 5.7 and $32 \mathrm{kHz}$ domains $(\boldsymbol{C}, \boldsymbol{D}) . \boldsymbol{E}, \boldsymbol{F}$, Relative position of all retrogradely labeled cells in $\mathrm{MGBv}(\boldsymbol{E})$ and $\mathrm{MGBm}(\boldsymbol{F})$ from low (green) and high (red) injections. Data points in foreground of scatterplots reflect mean rostral-caudal and lateral-medial positions \pm SEMs. G, Schematic of proposed tonotopic gradient with MGBv. Warmer colors represent higher-frequency BFs. V, MGBv; M, MGBm; D, dorsal division of MGB; CP, caudal pole; Sg, suprageniculate nucleus; R, rostral; M, medial; L, lateral. Scale bar, 0.25 mm.

made at a well tuned high-frequency site $0.13 \mathrm{~mm}$ lateral to the high-frequency reversal (Fig. $4 D$, top), and a medial lesion was made at the deepest site with tone-evoked activity, $0.21 \mathrm{~mm}$ medial to the high-frequency reversal point (Fig. $4 D$, bottom, $E$, white crosses). Localization of lesions in coronal sections reacted for cytochrome oxidase revealed that the recording site lateral to the $\mathrm{BF}$ reversal was in $\mathrm{MGBv}$ and the lesion medial to the $\mathrm{BF}$ reversal straddled the border between $\mathrm{MGBm}$ and the posterior thalamic complex (Fig. 4C). Indeed, in 13 of 14 lesions, the BF reversal correctly predicted the anatomical boundary dividing MGBv from MGBm (Fig. 4E).

\section{Tonotopy in MGBv and MGBm}

Using the high-frequency reversal point as a neurophysiological marker for the boundary between the ventral and medial divisions, we were able to rigorously test each of the three predictions enumerated above. The BF reversal point was identified in each penetration (Fig. 5A, black square), and the slope of the linear fit lines was calculated independently from the rising and falling components of the BF functions (Fig. 5A). Returning to the first prediction, BFs measured along the lateral wall of the MGBv were found to decrease significantly along the rostral-to-caudal extent $(N=43$, one-way ANOVA, $F=4.67$, $R^{2}=0.96, p<0.05$ ) (Fig. $5 B$ ). For the second prediction, we found that BF increased significantly from lateral to medial positions within $\mathrm{MGBv}(F=26.1$, $p<1 \times 10^{-6}$ ) (Fig. 5C, gray circles), and the slope of the increasing BF function did not differ according to where recordings were made within the rostral-to-caudal extent of $\operatorname{MGBv}(F=1.0, p=0.55)$ (Fig. $5 D$, gray circles).

With regard to the third prediction, despite the extensive comingling of lowand high-frequency AI projection neurons within the MGBm, neurophysiological recordings indicated that $\mathrm{BF}$ decreased significantly across the lateral-to-medial extent of the $\operatorname{MGBm}(F=2.75, p<0.005)$ (Fig. $5 C$, black squares), and this negative slope did not vary systematically across the rostral-to-caudal extent of the MGBm $(F=0.41, p=0.97)$ (Fig. 5D, black squares). Therefore, tonotopy in MGBv closely matched our predictions from the retrograde tracer studies, yet we also found evidence for a mirror reversal decreasing BF gradient within MGBm, which was not predicted by the anatomical data.

\section{Variations in spectral bandwidth and onset latency in auditory cortex and MGB}

Analysis of response properties at individual recording sites proved to be another useful tool for distinguishing AI vs AAF and MGBv vs MGBm. FRA bandwidth measured $10 \mathrm{~dB}$ above threshold was significantly broader in AAF than AI $(0.82 \pm 0.03$ vs $0.92 \pm 0.04$ octaves; unpaired $t$ test, $p<0.05)$ and in MGBm compared with $\operatorname{MGBv}(1.12 \pm 0.04$ vs $0.89 \pm 0.03$ octaves; unpaired $t$ test, $p<$ $\left.1 \times 10^{-6}\right)$ (Fig. $5 E$ ). Consistent with previous observations in mouse (Linden et al., 2003) and rat (Polley et al., 2007), we found that tone-evoked onset latency was significantly shorter in AAF than AI (12.39 \pm 0.17 vs $14.79 \pm 0.3 \mathrm{~ms}$; unpaired $t$ test, $p<$ $0.0001)$. Interestingly, onset latencies decreased by $5.3 \mathrm{~ms}$ across the $\mathrm{BF}$ range in $\mathrm{AI}$ (one-way ANOVA, $F=5.98, p<5 \times 10^{-5}$ ) (Fig. $5 F)$, but not in $\operatorname{AAF}(F=1.32, p=0.26)$. Similarly, onset latency was significantly shorter on average in MGBm than MGBv (9.75 \pm 0.17 vs $10.75 \pm 0.18 \mathrm{~ms}$; unpaired $t$ test, $p<$ $0.0001)$ and decreased by $5.3 \mathrm{~ms}$ across the BF range in MGBv $\left(F=24.98, p<1 \times 10^{-6}\right)$ without varying significantly in $\operatorname{MGBm}(F=0.59, p=0.7)$ (Fig. $5 F)$. Note that the latency shift observed between high and low BF recording sites in $\mathrm{AI}$ and $\mathrm{MGBv}$ cannot be accounted for by the basilar membrane group 
delay. Tone-evoked onset spikes recorded in single nerve fibers from the basal (highfrequency) versus apical (low-frequency) regions of the mouse cochlea occur at $1.86 \pm 0.07$ and $2.89 \pm 0.11 \mathrm{~ms}$, respectively, which can only account for 20\% of the shift observed in $\mathrm{AI}$ and $\mathrm{MGBv}$ (M. C. Liberman, personal communication) (Fig. 5F, black squares).

Topographic mapping in the thalamocortical slice

Using the BF gradients characterized in AI and MGBv of the intact mouse, we turned to the acute thalamocortical slice preparation to determine whether tonotopic organization corresponded to topographic connectivity. Specifically, we predicted that electrical stimulation of the MGBv along the caudolateral-to-rostromedial tonotopic gradient that emerged from tracer and mapping studies would yield a smooth shift in AI activity foci along the caudal-to-rostral, low-to-high BF gradient established from our cortical mapping studies. This prediction could be effectively tested through VSD imaging in the thalamocortical slice by applying a discrete stimulus to the MGBv and measuring subthreshold VSD signal changes across the entire caudal-to-rostral extent of AI simultaneously. VSD signal amplitude was measured across 18 ROIs $(125 \times$ $125 \mu \mathrm{m}$ each) positioned either in layer IV, where VSD response amplitudes were greatest, or in an immediately dorsal region in layer II/III.

In support of our prediction, we observed that rostral areas of AI were most responsive to rostromedial MGBv stimulation (high BFs); whereas, more caudal areas of the AI map were more effectively activated by caudolateral MGBv stimulation (low/mid BFs) (Fig. 6A). Stimulation of MGBv at six loci along the optimized caudolateral-to-rostromedial

orientation-induced, broad, overlapping, subthreshold activation profiles that nevertheless shifted significantly across the caudal-to-rostral extent of layer IV in AI (mean \pm SEM ROI for site 1 vs 6, 7.6 \pm 0.8 vs $12.8 \pm 0.8$; $t$ test, $p<0.0001$ ) (Fig. $6 B$ ). Average AI layer IV responses were normalized for each stimulation site and represented as a color map, with the upward diagonal band representing the point-topoint topographic mapping between $\mathrm{MGBv}$ and AI (Fig. 6C). Topographic shifts along the caudal-to-rostral axis were specific to the optimized stimulation orientation, as stimulating six loci along an orthogonal orientation did not significantly shift the activity profiles, consistent with the expected effects of stimulating the MGBv along an isofrequency contour $(9.2 \pm 1.9$ vs $10.0 \pm$
A

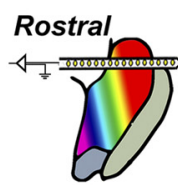

Intermediate
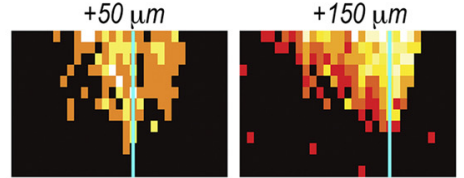

$+150 \mu m$

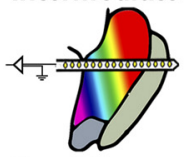

caudal

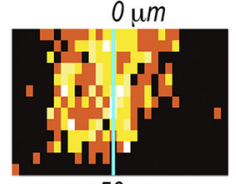

$+50 \mu m$

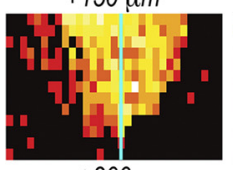

$+300 \mu m$
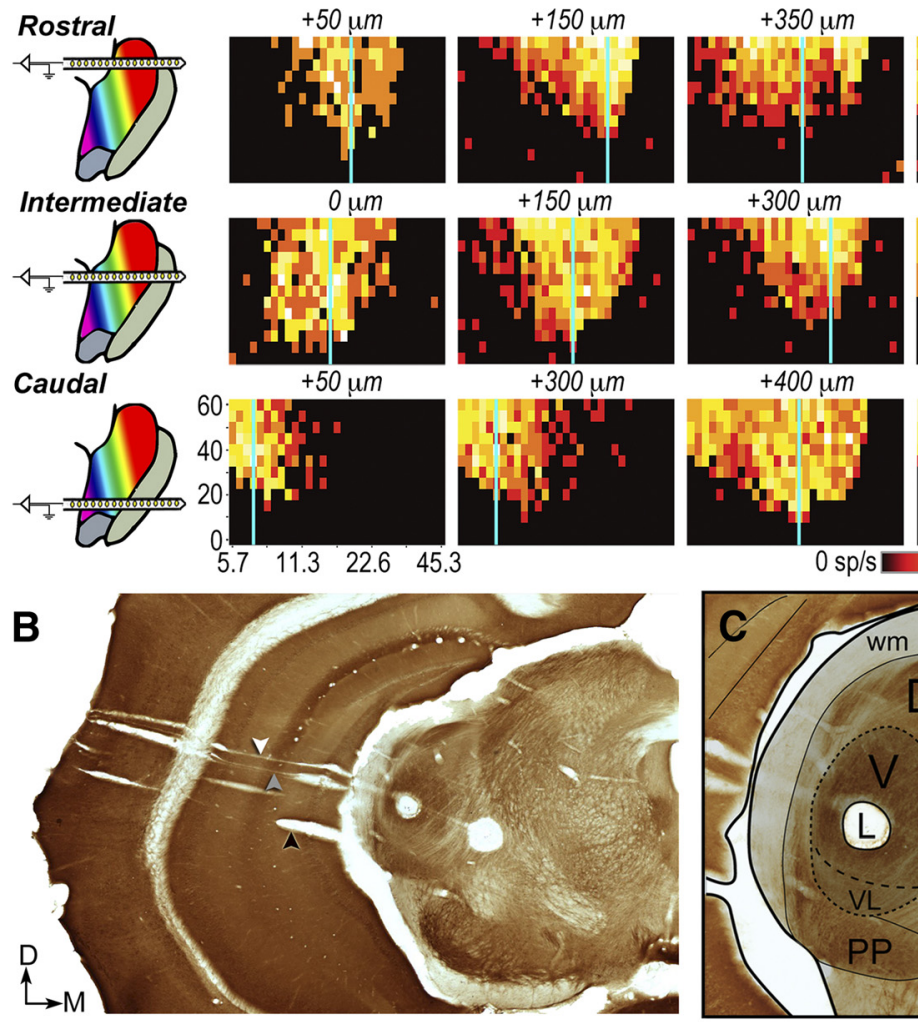

$+300 \mu m$

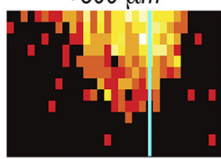

$+400 \mu m$

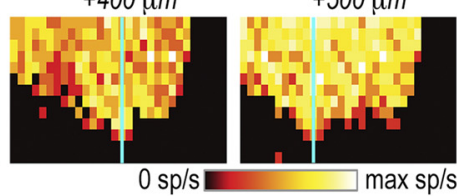

$0 \mathrm{sp} / \mathrm{s} \square \max \mathrm{sp} / \mathrm{s}$

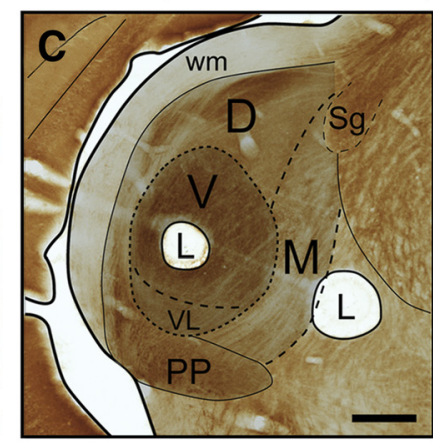

D

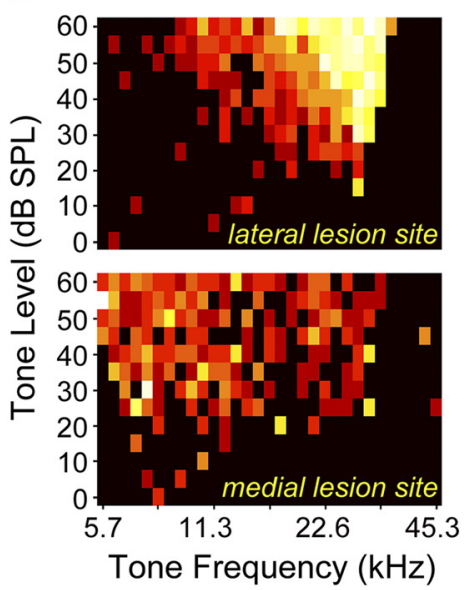

E

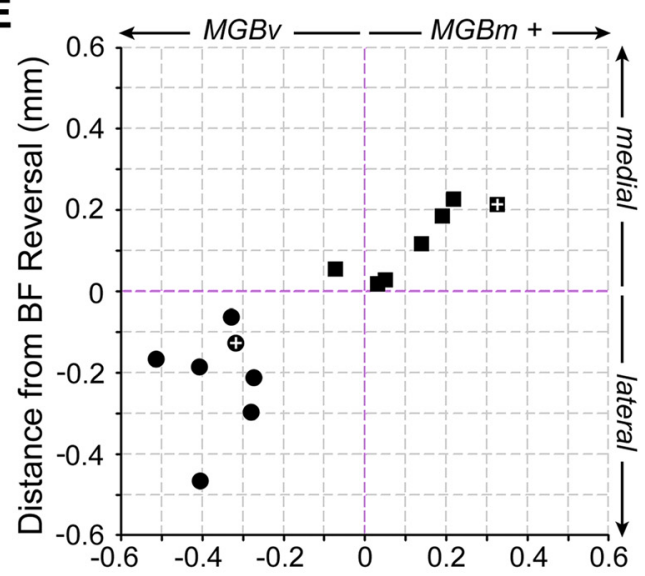

Distance from Anatomical Boundary ( $\mathrm{mm}$ )

Figure 4. Neurophysiological dissociation of MGBv from MGBm. $\boldsymbol{A}$, Recordings were made along the lateral-to-medial extent of the MGB (across columns) using a multichannel silicon probe inserted along the same plane used for the thalamocortical slice. Multiple penetrations were made in each mouse to permit comparison of recordings made across the rostral-to-caudal extent of the MGB (across rows). Distance from the lateral wall of the MGBv is stated for FRA. Vertical blue line represents BF. $\boldsymbol{B}, \boldsymbol{C}$, low- and high-power images of a coronal section reacted for cytochrome oxidase. The black arrow indicates the initial probe insertion to locate the ventral margin of the MGB (see Materials and Methods), the gray arrow indicates the track made by the silicon probe penetration used for recordings, and the white arrow represents the electrode track used to create the lesions. $\boldsymbol{C}$, High-power image depicts the location of two lesions $(\mathrm{L})$ drawn relative to neighboring anatomical landmarks. $D$, FRAs measured immediately before lesioning at the lateral and medial lesion sites shown in $\boldsymbol{B}$ and $\boldsymbol{C}$. $\boldsymbol{E}$, The location of lesions made lateral or medial to the $B F$ reversal point (circles and squares, respectively) are shown relative to the anatomical boundary dividing MGBv from MGBm, along with the electrode travel distance from the frequency reversal. MGBm + indicates that data points could have been drawn from auditory-responsive sites medial to the MGBm. White crosses represent the lesions shown in $B$ and $\boldsymbol{C}$. sp/s, Spikes per second; PP, peripeduncular nucleus; wm, white matter. Scale bar, $0.25 \mathrm{~mm}$.

$1.1 ; p=0.72$ ) (Fig. $6 D, E$ ). As a final test, we returned to the optimized orientation but shifted the ROIs to layer II/III, rather than layer IV. Topographic shifts in layer II/III were weak, but not significant $(7.6 \pm 0.9$ vs $10.4 \pm 1.4 ; p=0.1$ ) (Fig. $6 F, G)$, in 
A
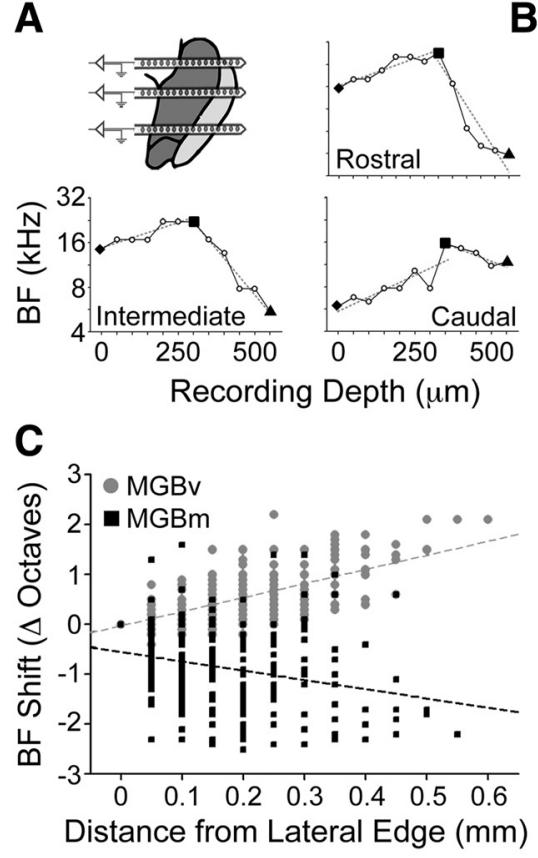

E

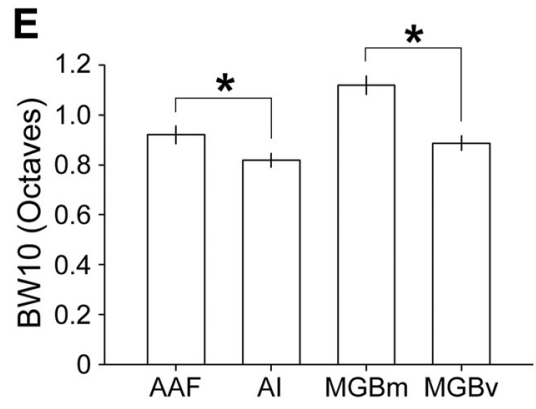

B

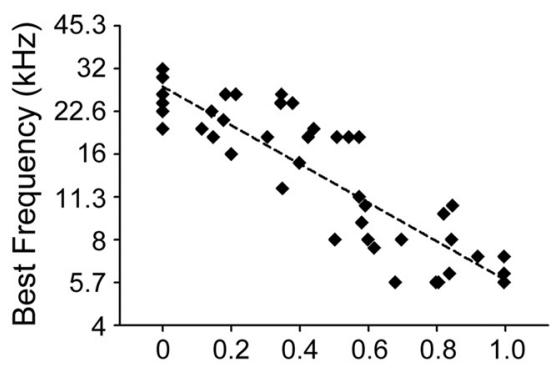

Norm. Rostral-to-Caudal Position

D

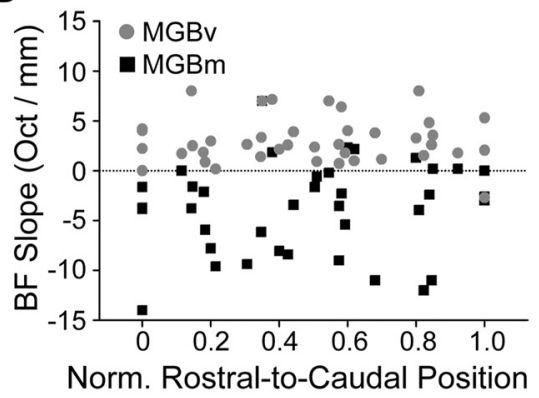

$\mathbf{F}$

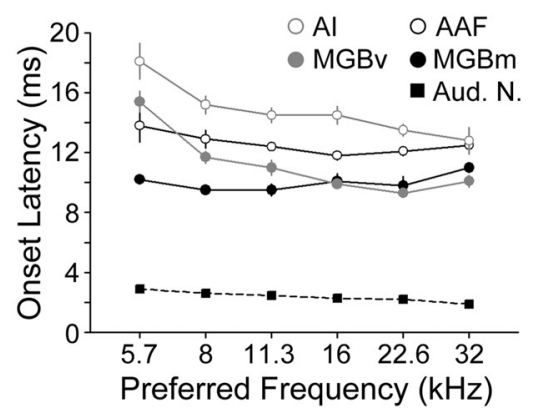

Figure 5. Tonotopic organization of MGBv and MGBm. $A$, Examples of nonmonotonic BF functions across the lateral-to-medial depth within MGB obtained from rostral, intermediate, and caudal positions. The high-frequency reversal was used to divide MGBv (diamond to square) from MGBm (square to triangle) recording sites. Dashed lines represent linear fits of MGBv and MGBm BF functions used to calculate $B F$ slope $(\boldsymbol{D}) . \boldsymbol{B}, \mathrm{BF}$ measurements across the rostral-to-caudal extent of the lateral wall of MGBv. Dashed line represents linear fit. C, BF changes across the lateral-to-medial extent of MGBv (gray circles) and MGBm (black squares). Dashed lines indicate linear fits applied to BF shift data from MGBv (gray) or MGBm (black). D, Slope of BF changes across the rostral-to-caudal extent of the MGB. Positive values indicate increasing BF functions; negative values indicate decreasing BF functions. $E$, Mean \pm SEM FRA bandwidth measured $10 \mathrm{~dB}$ above the minimum response threshold. Asterisks indicate statistically significant differences using an unpaired $t$ test $(p<0.05) . \boldsymbol{F}$, Mean \pm SEM first spike latency as a function of $B F$ for MGB and cortex and characteristic frequency for auditory nerve fiber recordings. Bins are 0.5 octaves wide beginning at the value indicated by the tick label. Norm., Normalized; Aud. N., auditory nerve.

agreement with recent reports of heterogeneous tuning in the upper layers of mouse AI (Bandyopadhyay et al., 2010; Broicher et al., 2010; Rothschild et al., 2010).

\section{Linking maps of preferred frequency to spatially distributed frequency representations}

In one view, VSD imaging data from layer IV are in close agreement with AI and MGBv mapping studies: moving the stimulating electrode across the caudolateral-to-rostromedial tonotopic map in MGBv yields an orderly progression of activity foci across the caudal-to-rostral tonotopic map in AI. This finding thus projects a basic sensory map onto the acute auditory thalamocortical slice preparation to enable high-resolution studies of synaptic physiology. However, from another perspective, the BF maps in vivo exhibited relatively precise point-to-point mapping between a tone frequency and its preferred representation in the cortical

map; whereas, stimulating a single point in MGBv-evoked VSD activity across the majority of the AI map. In other words, positional shifts in peak VSD signals corroborate the in vivo mapping of AI, but the breadth and overlap of the VSD activity profiles do not. Why might this be?

The fact that VSD is sensitive to subthreshold changes in membrane potential, while microelectrode mapping reflects spiking activity can certainly explain some of the differences between the two datasets (Berger et al., 2007). On the other hand, the discrepancy may also be attributed to the nature of BF maps, which only represent the single preferred frequency for a given recording site, compared with VSD activation maps, which measure the entire spatial spread of population activity evoked by a point stimulus.

To address this difference in conceptual approach and to reconcile the basic differences in the degree of tonotopic mapping precision with these two methods, we reanalyzed our in vivo recordings according to the spatial spread of toneevoked excitation rather than preferred frequency. Returning to the same mapping experiment shown in Figure 1, we surveyed which recording sites contained 8.6 and $19.7 \mathrm{kHz}$ tones presented at $50 \mathrm{~dB}$ SPL within their FRAs (Fig. 7A). Compared with Figure $1 A$, one can appreciate that tone representations in AI appeared less spatially focused than the orderly BF map, and tone representations in $\mathrm{AAF}$ were even broader and more overlapping.

To facilitate direct comparison with the VSD imaging data, we grouped recording sites from each AI and AAF map into $18 \mathrm{ROIs}$ that spanned the tonotopic gradient in each field. We then selected six tone frequencies homologous to the six MGBv stimulation sites and determined the probability that neurons in each ROI would contain a given $50 \mathrm{~dB}$ SPL tone frequency within their FRA. Spiking probability profiles in AI were broader than the BF preference maps, but not as broad as the subthreshold VSD maps (Fig. $7 B$ ). Tones of different frequency activated tonotopically appropriate regions within $\mathrm{AI}$, yet the response profiles were quite broad, with frequencies $<20 \mathrm{kHz}$ activating approximately half of the map with $\mathrm{a} \geq 0.5$ probability.

Normalized color maps were created in a similar fashion to those presented in Figure 6 and further demonstrated that spatial representations of tone frequencies in AI were broad, but spatially organized with a narrow upward diagonal band that was fairly stable across tone levels (Fig. 7E). Compared with AI, spatial representations of tones in AAF were degraded and level intolerant (Fig. 7C,F), which was to be expected given the greater BF scatter (Fig. 1C,D), compressed range of tone frequency representation (Fig. 1C,D), and broader frequency tuning (Fig. $5 E$ ). We also investigated spatial activation profiles in the $\mathrm{MGBv}$ 

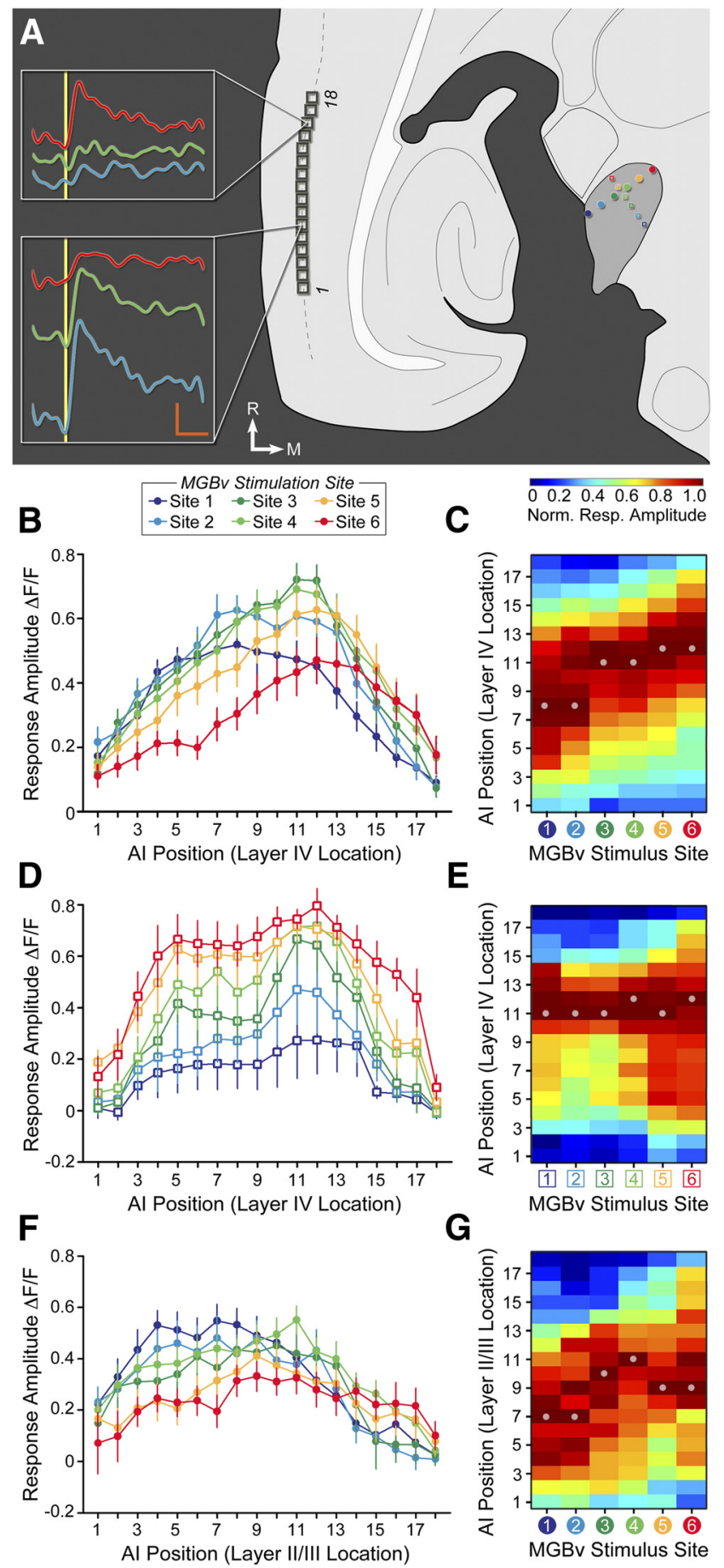

Figure 6. Topographic mapping in the thalamocortical slice. $\boldsymbol{A}$, Thalamocortical slice schematic represents the orientation of the six MGBv stimulation sites used for optimized (filled circles) and orthogonal (open squares) comparisons as well as 18 ROls positioned atop layer IV (dashed gray line). Insets, Representative VSD signals evoked from ROI 16 or 6 from a $1 \mathrm{~m}$ current pulse (vertical yellow bar) to the color-matched region of MGBv. Vertical orange bar, $0.03 \%$ fractional change in signal strength; horizontal orange bar, 0.1 s. $\boldsymbol{B}-\mathbf{G}$, Fractional change in VSD response amplitudes across the $18 \mathrm{RO}$ Is positioned in layer IV for the optimized stimulus orientation $(\boldsymbol{B}, \boldsymbol{C})$ and orthogonal orientation $(\boldsymbol{D}, \boldsymbol{E})$, and in layer II/III for the optimized trajectory $(\boldsymbol{F}, \boldsymbol{G}) . \boldsymbol{C}, \boldsymbol{E}, \boldsymbol{G}$, Surface plots represent fractional change in VSD response amplitudes that have been normalized for each stimulation site. For optimized stimulation orientation, site 1 is caudolateral and site 6 is rostromedial. For orthogonal stimulation, site 1 is caudomedial and site 6 is rostrolateral. Dots indicate location of peak cortical VSD signal. Norm., Normalized; Resp., response. across nine ROIs positioned along the caudolateral-torostromedial BF gradient. Qualitatively, the spatial code for tone frequency representation in MGBv was less organized and less level tolerant than AI, but superior to AAF (Fig. 7D, G).

Spatial activation profiles were further quantified in $\mathrm{AI}$ on an individual mouse basis by measuring the peak and width at half maximum of the normalized response functions for each frequency summed across 30-60 dB SPL. We observed significant spatial shifts in the caudal and rostral boundaries at half maximum (Friedman nonparametric ANOVA, $p<0.005$ for both) as well as the peak position $(p<0.01)$ with tones of increasing frequency (Fig. $7 H$ ). Overall, each tone activated one-third to one-half of AI ROIs. As a final step, we directly compared spiking half-height boundaries with the normalized VSD half-height boundaries and the average BF values across all ROIs (Fig. 7I).

The upward diagonal slant present in all three measures demonstrates that layer IV of AI is tonotopically organized regardless of whether the input signal to the mapping function is subthreshold VSD, suprathreshold spiking, or BF. However, the precision of the layer IV mapping function varies between techniques. Subthreshold activity profiles suggested a coarse mapping specificity wherein any point in the MGBv map can activate nearly any point in the AI map. Suprathreshold spiking patterns produce a much higher degree of mapping precision, wherein a given tone can recruit activity across a swath of the AI map, yet only a small cluster of neurons within this active zone will claim this tone as the BF.

\section{Discussion}

The aims of these experiments were to resolve the discrepancy surrounding the existence of tonotopy in the mouse auditory cortex, to provide the first evidence for or against the existence and orientation of a tonotopic map within the mouse MGB, and to determine whether the tonotopic organization in both brain areas could be captured within the thalamocortical slice in vitro. We determined that the middle layers of AI exhibited a clear tonotopic organization with BFs ranging from 5.6 to $\sim 34 \mathrm{kHz}$ between the caudal and rostral boundaries, respectively. Retrograde tracer injections into low and high BF domains in AI revealed topographically separable MGBv projections combined with a weaker, topographically mixed projection from MGBm. The relative position of retrogradely labeled cell bodies suggested a low-to-high, caudolateral-to-rostromedial BF organization in MBGv, which was subsequently corroborated with dense twodimensional receptive field mapping. Stimulating the thalamocortical slice along the same tonotopic gradient in $\mathrm{MGBv}$ confirmed the expected caudal-to-rostral shift in VSD activity foci along the AI tonotopic gradient in layer IV (Fig. 8A).

\section{Toward a cellular analysis of regional specialization in the auditory thalamocortical circuit}

The combination of in vivo and in vitro approaches will facilitate detailed cellular analyses of the mechanisms supporting specialized auditory signal processing within subdomains of the tonotopic map. For example, do variations in first spike latency and tuning bandwidth across the tonotopic gradient reflect differences in local circuit properties or cell-autonomous regulation of dendritic morphology, ligand-gated or voltage-gated ion channels, as have been reported in the auditory brainstem nuclei (Smith and Rubel, 1979; Sanes et al., 1987; Li et al., 2001; Sanchez et al., 2010; Strumbos et al., 2010)? The spatial extent of a tone representation within the $\mathrm{AI}$ or MGB tonotopic map can also be modified through learning, trauma, or normal age-related pro- 


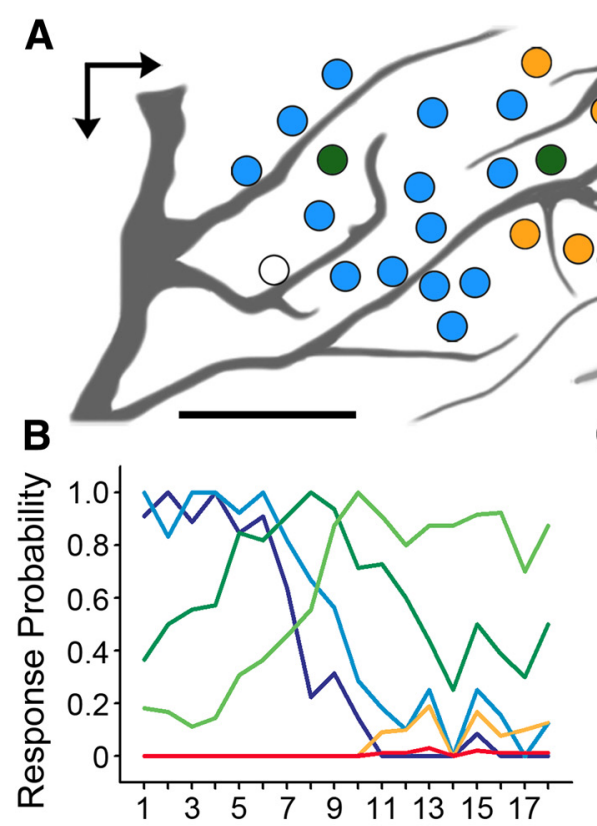

Al Position (Caudal to Rostral)

E

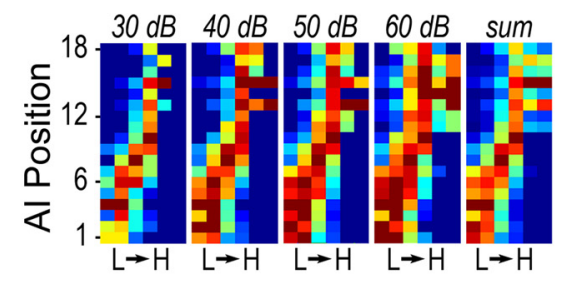

Tone Frequency (5.7 to $36.8 \mathrm{kHz}$ )
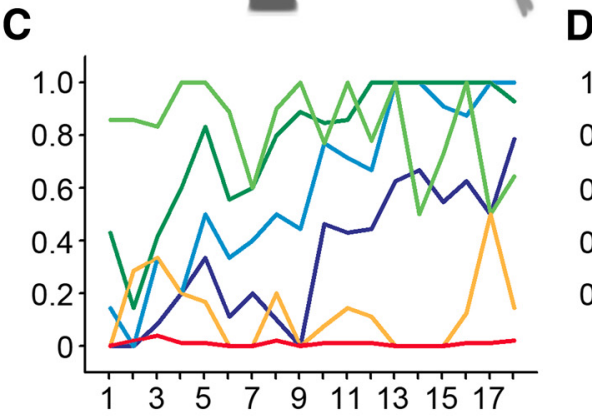

AAF Position (Caudal to Rostral)
$\mathbf{F}$

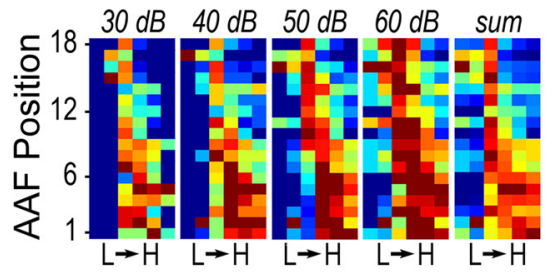

H

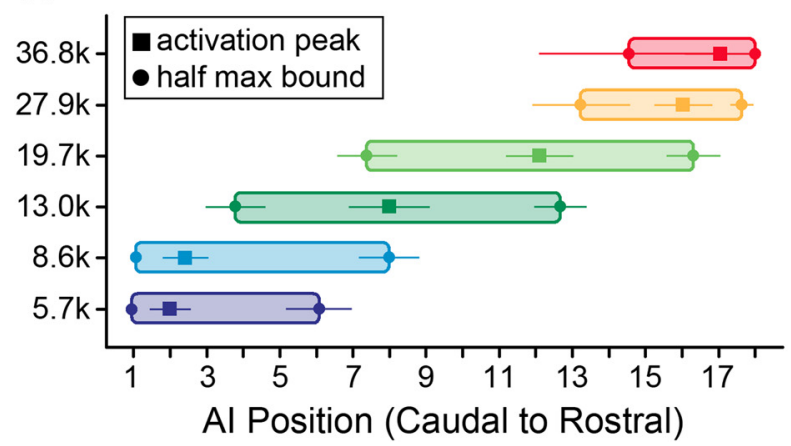

I

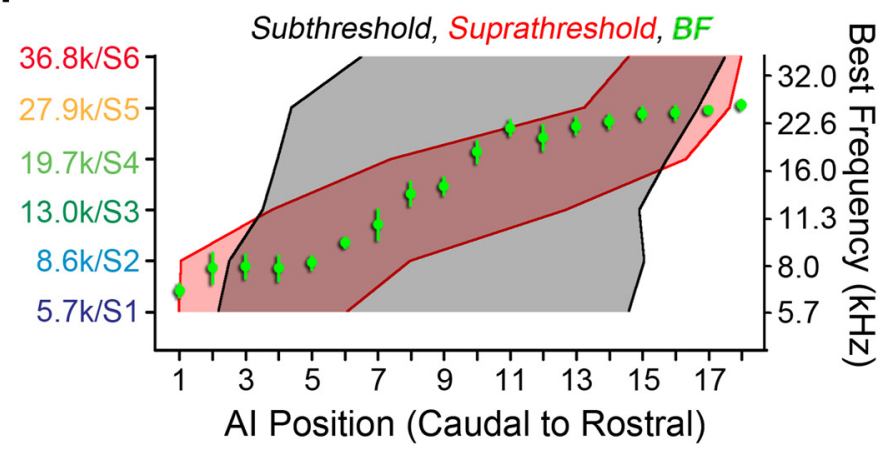

Figure 7. Analysis of spatially distributed tone representations. $A$, Color of the same Al (circles) and AAF (squares) recording sites shown in Figure 1 represents whether they contained 8.6 and/or $19.7 \mathrm{kHz}$ tones at $50 \mathrm{~dB}$ SPL in their FRA. $\boldsymbol{B}-\boldsymbol{D}, \mathrm{AI}(\boldsymbol{B})$ and $\mathrm{AAF}(\boldsymbol{C})$ recording sites were divided into 18 ROls ordered by their caudal-to-rostral position; MGBv recording sites ( $\boldsymbol{D})$ were grouped into 9 ROIs according to their location along the caudolateral-to-rostromedial ( $C L$ and RM, respectively) axis. Line plots represent the probability that recording sites within each ROI contain each of six different $50 \mathrm{~dB}$ SPL tones in their FRA. $\boldsymbol{E}-\mathbf{G}$, Surface plots represent the relative probability that recording sites in each ROI will contain the same six frequencies ranging from low $(\mathrm{L})$ to high $(\mathrm{H})$ in their FRA when presented at various sound levels or the summed probability across all sound levels in $\operatorname{AI}(\boldsymbol{E}), \operatorname{AAF}(\boldsymbol{F})$, and MGBv $(\boldsymbol{G})$. $\boldsymbol{H}, \mathrm{Mean} \pm \mathrm{SEM}$ ROI position of normalized probability peak (square) and the half-height boundaries measured caudal and rostral to the peak for each stimulation frequency in AI. Data are derived from the sum of sound levels. $I$, Mean caudal and rostral half-height response boundaries derived from AI VSD maps (black) and Al normalized response probability functions (red). Mean \pm SEM BF within each ROI is superimposed (green). Norm., Normalized; Resp., response; S, MGBv stimulation site.

cesses (for review, see Dahmen and King, 2007). It would be interesting to explore whether tonotopic remapping induced by high-frequency age-related hearing loss or focal lesions of the basilar membrane arise through regionally restricted alterations in excitatory-inhibitory balance, consistent with the global dysregulation following pervasive sensorineural hearing loss (Rob- ertson and Irvine, 1989; Willott et al., 1993; Kamke et al., 2003; Kotak et al., 2005, 2008).

Similarly, numerous studies have found that passively rearing young animals in ambient sound environments dominated by a single tone frequency, or conditioning adult animals to associate a neutral tone frequency with a behaviorally relevant outcome, 
induces large-scale reorganization of the AI tonotopic map (Polley et al., 2006; de Villers-Sidani et al., 2007; Dorrn et al., 2010). Developmentally and spatially specific changes of intracellular and synaptic signaling that permit this reorganization in an otherwise stable map remain unclear. By establishing a tonotopic map in the thalamocortical slice, we can begin to explore how differences in cell morphology, gene expression, synaptic dynamics, and intrinsic physiology may give rise to regional specializations in the representation and plasticity of auditory stimuli.

\section{What is a tonotopic map?}

The history of tonotopic mapping studies in $\mathrm{AI}$ is reminiscent of the Indian parable of the three blind men who each examine a different part of an elephant's body with their hands (e.g., the trunk, a leg, and the tail). Each man infers the appearance of the entire elephant based on their restricted experience, but upon comparison discovers that his view is highly divergent and mutually incompatible with the others. The presence or absence of a tonotopic organization in AI has been in dispute since the earliest published accounts of electrophysiological recordings in the auditory cortex. Some initial studies reported that AI is tonotopically organized due to the similarity of BFs within a single vertical penetration and the smooth systematic variation of BF across the expanse of AI (Woolsey and Walzl, 1942; Tunturi, 1962; Merzenich and Brugge, 1973), while others have found that tonotopic organization in AI is weak or nonexistent based on observations that BFs could vary considerably within a single penetration (Evans et al., 1965) with no discernable gradient across AI (Evans and Whitfield, 1964; Goldstein and Abeles, 1975).

Contradictory findings continue to the present day, with a pair of elegant in vivo calcium imaging studies reporting that BFs between neighboring neurons in superficial layers of mouse AI can vary by several octaves and form a very coarse tonotopic organization only when compared over the entire extent of AI (Bandyopadhyay et al., 2010; Rothschild et al., 2010). The authors of these studies suggest that earlier reports of finescale tonotopy could be attributed to the comparatively coarse spatial sampling used by microelectrode mapping studies (at $100-200 \mu \mathrm{m}$ intervals), pooling of heterogeneous local tuning through multiunit recording and a selection bias toward responsive recording sites. However, the fine-scale tonotopy reported here was acquired with higher spatial resolution (25 $\mu \mathrm{m}$ sampling density along the tonotopic axis) and would be virtually impossible to obtain through a random selection of locally heterogeneous tuning. Moreover, the electrode tip was inserted into the thalamorecipient layers ( $420-440 \mu \mathrm{m}$ beneath the pia) without any systematic fine tuning to find auditory-driven sites. Last, even though AI FRAs were derived from multiunit clusters, the tuning

B
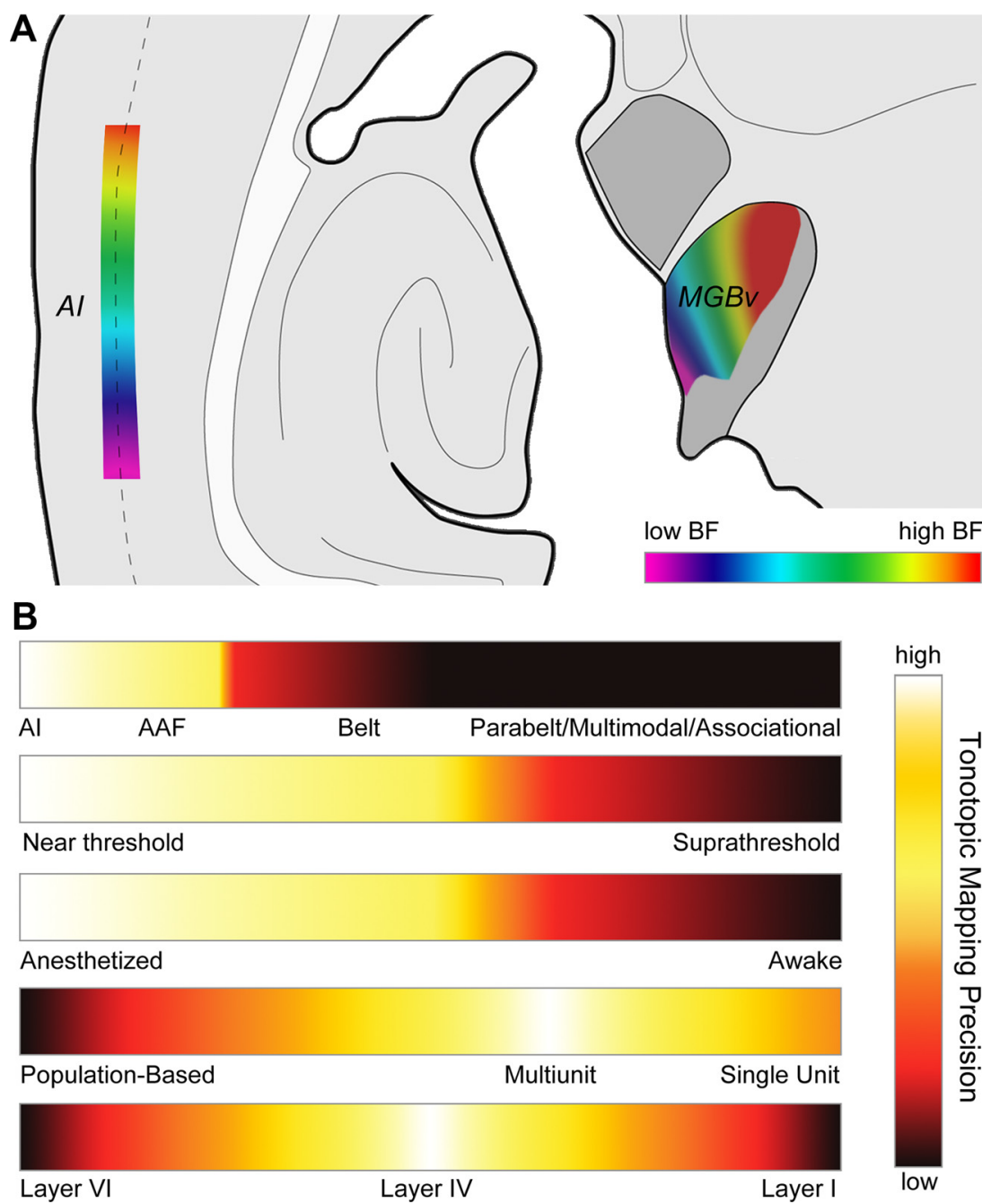

Figure 8. Implications for future studies of tonotopy in the thalamocortical circuit. $\boldsymbol{A}$, Superposition of tonotopic gradients erization of preferred tuning, the state of vigilance, the spatial resolution of the measurement, and the cortical layer from which recordings are made (from top to bottom).

was considerably narrower and more $\mathrm{V}$ shaped than what would be expected from the combination of scattered local tuning described in the imaging studies. The precisely organized neurophysiological maps and topographically organized anatomical projections from MGBv leave no doubt that mouse AI is tonotopically organized.

Much like the story of the blind men and the elephant, we would argue that the interpretations of both datasets are individually correct but ultimately fail to adequately describe the whole. In our own experiments, we found that tonotopic precision was considerably degraded when maps were derived from a population-based suprathreshold spiking analysis (Fig. 7), even further blurred when assessed with subthreshold VSD signals in layer IV (Fig. 6B, C) and not statistically significant based upon VSD measurements from layer II/III (Fig. 6F, G). It is our view, therefore, that AI is both tonotopically and nontonotopically organized, depending on the manner in which it is studied.

We would argue that tonotopic mapping precision depends on the following five principle factors: (1) whether recordings were made within AI versus other fields within the core, belt, parabelt/multimodal heterarchy (Hackett, 2011); (2) sound level used for the preferred frequency characterization; (3) anesthetic 
state; (4) spatial resolution of the measurement technique; and (5) laminae in which measurements are made (Fig. $8 \mathrm{~B}$ ). Studies that characterize preferred frequency from multiunit recordings in the middle layers of AI in barbiturate-anesthetized animals with near-threshold stimuli invariably find a high degree of tonotopic precision. Studies that deviate from this combination by, for example, recording calcium changes from isolated single units in the superficial layers of awake animals or measuring blood oxygenation-level dependent signals from awake subjects with a single high-intensity sound level are less likely to observe a tonotopic map.

In this scheme, tonotopically organized BF maps in AI, which predominantly reflect topographically organized MGBv projections into layers IIIb and IV, are the "tip of the iceberg" for frequency selectivity (Figs. 2, 3) (Winer et al., 1999; Lee et al., 2004; Budinger and Scheich, 2009). MGBv afferents provide the principal source of subcortical input to the developmentally mature AI (Romanski and LeDoux, 1993; de Venecia et al., 1998; Cetas et al., 1999; Huang and Winer, 2000; Kimura et al., 2003) by forming efficacious excitatory synapses onto stubby dendritic spines within $100 \mu \mathrm{m}$ of the AI cell bodies (Richardson et al., 2009). The present and prior studies have shown that MGBv neurons project to isofrequency regions of the AI map and that AI neurons in layer IIIb/IV inherit their BF from a focal convergence of MGBv neurons with frequency preferences that fall within one-third of an octave (i.e., one critical band) (Imig and Morel, 1984; Winer et al., 1999). However, AI frequency preference can also reflect less tonotopically organized inputs arising from local and long-range corticocortical connections (Kaur et al., 2004, 2005; Liu et al., 2007; Lee and Sherman, 2008; Wu et al., 2008; Happel et al., 2010; Moeller et al., 2010). The influence of these sources on cortical frequency tuning may be accentuated when recordings are made from the upper cortical layers (i.e., the source of $\mathrm{Ca}^{2+}$ imaging signals), where response latencies are longer, spectrotemporal receptive fields more complex and labile, and horizontal corticocortical connections more abundant (Barth and Di, 1990; Ojima et al., 1991; Wallace et al., 1991; Kaur et al., 2005; Barbour and Callaway, 2008; Dahmen et al., 2008; Atencio and Schreiner, 2010a,b).

As suggested from the slice data presented here and demonstrated through whole-cell recordings in the intact animal, the range of tone-evoked subthreshold inputs is substantially broader than the spiking receptive field (Wehr and Zador, 2003; Zhang et al., 2003; Kaur et al., 2004). These unexpressed synaptic inputs provide a latent substrate for remodeling the frequency preference of AI neurons, changes that can occur within minutes and last for many hours to days (Bakin and Weinberger, 1990; Fritz et al., 2003; Froemke et al., 2007; Dorrn et al., 2010). Similarly, recordings made under anesthesia, particularly in the upper layers, are likely to significantly underestimate modulatory inputs that arise from local circuits and higher cortical areas and overexaggerate the normally limited contribution of tonotopically organized MGBv inputs (Kuhn et al., 2008; Fritz et al., 2010; Sadagopan and Wang, 2010). The diverse and dynamic set of potential input sources interacting within and between cortical columns in the unanesthetized cortex provides a means for neighboring neurons to assume substantially different, yet sharply tuned frequency preferences. Thus, the same set of AI neurons could be both tonotopically and nontonotopically organized, depending on the source of synaptic inputs that dominate under a given experimental approach.

Although tonotopically organized inputs can be de-emphasized in some cortical operating modes, it would be incorrect to conclude that topographically organized feature representations are merely an artifact of anesthesia and are unworthy of further study. Regionally specialized cortical modules in AI can highlight areas with strong mutual connectivity (Matsubara and Phillips, 1988; Read et al., 2001), specialized afferent input from the MGB (Middlebrooks and Zook, 1983; de la Mothe et al., 2006; Read et al., 2008), or precise corticofugal connections with neurons in the thalamus (Zhang and Yan, 2008), midbrain (Zhang et al., 1997), brainstem (Luo et al., 2008), and even indirectly to outer hair cells (Xiao and Suga, 2002). Furthermore, reorganization of cortical spatial maps and modules can be a sensitive index for developmental critical periods (Insanally et al., 2009; Popescu and Polley, 2010), perceptual learning (Polley et al., 2006), or functional status of the auditory periphery (Irvine and Rajan, 1996). Therefore, a deep understanding of AI circuitry will likely require a synthesis of complementary approaches.

\section{References}

Atencio CA, Schreiner CE (2010a) Laminar diversity of dynamic sound processing in cat primary auditory cortex. J Neurophysiol 103:192-205.

Atencio CA, Schreiner CE (2010b) Columnar connectivity and laminar processing in cat primary auditory cortex. PLoS One 5:e9521.

Bakin JS, Weinberger NM (1990) Classical conditioning induces CSspecific receptive field plasticity in the auditory cortex of the guinea pig. Brain Res 536:271-286.

Bandyopadhyay S, Shamma SA, Kanold PO (2010) Dichotomy of functional organization in the mouse auditory cortex. Nat Neurosci 13:361-368.

Barbour DL, Callaway EM (2008) Excitatory local connections of superficial neurons in rat auditory cortex. J Neurosci 28:11174-11185.

Barth DS, Di S (1990) Three-dimensional analysis of auditory-evoked potentials in rat neocortex. J Neurophysiol 64:1527-1536.

Bendor D, Wang X (2010) Neural coding of periodicity in marmoset auditory cortex. J Neurophysiol 103:1809-1822.

Berger T, Borgdorff A, Crochet S, Neubauer FB, Lefort S, Fauvet B, Ferezou I, Carleton A, Lüscher HR, Petersen CC (2007) Combined voltage and calcium epifluorescence imaging in vitro and in vivo reveals subthreshold and suprathreshold dynamics of mouse barrel cortex. J Neurophysiol 97:3751-3762.

Broicher T, Bidmon HJ, Kamuf B, Coulon P, Gorji A, Pape HC, Speckmann EJ, Budde T (2010) Thalamic afferent activation of supragranular layers in auditory cortex in vitro: a voltage sensitive dye study. Neuroscience 165:371-385.

Budinger E, Scheich H (2009) Anatomical connections suitable for the direct processing of neuronal information of different modalities via the rodent primary auditory cortex. Hear Res 258:16-27.

Cetas JS, de Venecia RK, McMullen NT (1999) Thalamocortical afferents of Lorente de No: medial geniculate axons that project to primary auditory cortex have collateral branches to layer I. Brain Res 830:203-208.

Cruikshank SJ, Rose HJ, Metherate R (2002) Auditory thalamocortical synaptic transmission in vitro. J Neurophysiol 87:361-384.

Cruikshank SJ, Urabe H, Nurmikko AV, Connors BW (2010) Pathwayspecific feedforward circuits between thalamus and neocortex revealed by selective optical stimulation of axons. Neuron 65:230-245.

Dahmen JC, King AJ (2007) Learning to hear: plasticity of auditory cortical processing. Curr Opin Neurobiol 17:456-464.

Dahmen JC, Hartley DE, King AJ (2008) Stimulus-timing-dependent plasticity of cortical frequency representation. J Neurosci 28:13629-13639.

de la Mothe LA, Blumell S, Kajikawa Y, Hackett TA (2006) Thalamic connections of the auditory cortex in marmoset monkeys: core and medial belt regions. J Comp Neurol 496:72-96.

de la Rocha J, Marchetti C, Schiff M, Reyes AD (2008) Linking the response properties of cells in auditory cortex with network architecture: cotuning versus lateral inhibition. J Neurosci 28:9151-9163.

de Venecia RK, Smelser CB, McMullen NT (1998) Parvalbumin is expressed in a reciprocal circuit linking the medial geniculate body and auditory neocortex in the rabbit. J Comp Neurol 400:349-362.

de Villers-Sidani E, Chang EF, Bao S, Merzenich MM (2007) Critical period window for spectral tuning defined in the primary auditory cortex (A1) in the rat. J Neurosci 27:180-189. 
Dorrn AL, Yuan K, Barker AJ, Schreiner CE, Froemke RC (2010) Developmental sensory experience balances cortical excitation and inhibition. Nature 465:932-936.

Evans EF, Whitfield IC (1964) Classification of unit responses in the auditory cortex of the unanaesthetized and unrestrained cat. J Physiol 171:476-493.

Evans EF, Ross HF, Whitfield IC (1965) The spatial distribution of unit characteristic frequency in the primary auditory cortex of the cat. J Physiol 179:238-247.

Fritz JB, David SV, Radtke-Schuller S, Yin P, Shamma SA (2010) Adaptive, behaviorally gated, persistent encoding of task-relevant auditory information in ferret frontal cortex. Nat Neurosci 13:1011-1019.

Fritz J, Shamma S, Elhilali M, Klein D (2003) Rapid task-related plasticity of spectrotemporal receptive fields in primary auditory cortex. Nat Neurosci 6:1216-1223.

Froemke RC, Merzenich MM, Schreiner CE (2007) A synaptic memory trace for cortical receptive field plasticity. Nature 450:425-429.

Godfrey DA, Kiang NY, Norris BE (1975) Single unit activity in the posteroventral cochlear nucleus of the cat. J Comp Neurol 162:247-268.

Goldstein MH Jr, Abeles M (1975) Note on tonotopic organization of primary auditory cortex in the cat. Brain Res 100:188-191.

Hackett TA (2011) Information flow in the auditory cortical network. Hear Res 271:133-146.

Hackett TA, de la Mothe LA (2009) Regional and laminar distribution of the vesicular glutamate transporter, VGluT2, in the macaque monkey auditory cortex. J Chem Neuroanat 38:106-116.

Happel MF, Jeschke M, Ohl FW (2010) Spectral integration in primary auditory cortex attributable to temporally precise convergence of thalamocortical and intracortical input. J Neurosci 30:11114-11127.

Huang CL, Winer JA (2000) Auditory thalamocortical projections in the cat: laminar and areal patterns of input. J Comp Neurol 427:302-331.

Imig TJ, Morel A (1984) Topographic and cytoarchitectonic organization of thalamic neurons related to their targets in low-, middle-, and highfrequency representations in cat auditory cortex. J Comp Neurol 227:511-539.

Insanally MN, Köver H, Kim H, Bao S (2009) Feature-dependent sensitive periods in the development of complex sound representation. J Neurosci 29:5456-5462.

Irvine DR, Rajan R (1996) Injury- and use-related plasticity in the primary sensory cortex of adult mammals: possible relationship to perceptual learning. Clin Exp Pharmacol Physiol 23:939-947.

Kaas JH (2011) The evolution of auditory cortex: the core areas. In: The auditory cortex (Winer JA, Schreiner CE, eds), pp 407-427. Berlin, Heidelberg, New York: Springer.

Kamke MR, Brown M, Irvine DR (2003) Plasticity in the tonotopic organization of the medial geniculate body in adult cats following restricted unilateral cochlear lesions. J Comp Neurol 459:355-367.

Kaur S, Lazar R, Metherate R (2004) Intracortical pathways determine breadth of subthreshold frequency receptive fields in primary auditory cortex. J Neurophysiol 91:2551-2567.

Kaur S, Rose HJ, Lazar R, Liang K, Metherate R (2005) Spectral integration in primary auditory cortex: laminar processing of afferent input, in vivo and in vitro. Neuroscience 134:1033-1045.

Kimura A, Donishi T, Sakoda T, Hazama M, Tamai Y (2003) Auditory thalamic nuclei projections to the temporal cortex in the rat. Neuroscience 117:1003-1016.

Kotak VC, Fujisawa S, Lee FA, Karthikeyan O, Aoki C, Sanes DH (2005) Hearing loss raises excitability in the auditory cortex. J Neurosci 25:3908-3918.

Kotak VC, Takesian AE, Sanes DH (2008) Hearing loss prevents the maturation of GABAergic transmission in the auditory cortex. Cereb Cortex 18:2098-2108.

Kuhn B, Denk W, Bruno RM (2008) In vivo two-photon voltage-sensitive dye imaging reveals top-down control of cortical layers 1 and 2 during wakefulness. Proc Natl Acad Sci U S A 105:7588-7593.

Lee CC, Sherman SM (2008) Synaptic properties of thalamic and intracortical inputs to layer 4 of the first- and higher-order cortical areas in the auditory and somatosensory systems. J Neurophysiol 100:317-326.

Lee CC, Imaizumi K, Schreiner CE, Winer JA (2004) Concurrent tonotopic processing streams in auditory cortex. Cereb Cortex 14:441-451.

Li W, Kaczmarek LK, Perney TM (2001) Localization of two high-threshold potassium channel subunits in the rat central auditory system. J Comp Neurol 437:196-218.

Linden JF, Liu RC, Sahani M, Schreiner CE, Merzenich MM (2003) Spectrotemporal structure of receptive fields in areas $\mathrm{AI}$ and $\mathrm{AAF}$ of mouse auditory cortex. J Neurophysiol 90:2660-2675.

Liu BH, Wu GK, Arbuckle R, Tao HW, Zhang LI (2007) Defining cortical frequency tuning with recurrent excitatory circuitry. Nat Neurosci 10:1594-1600.

Llano DA, Sherman SM (2009) Differences in intrinsic properties and local network connectivity of identified layer 5 and layer 6 adult mouse auditory corticothalamic neurons support a dual corticothalamic projection hypothesis. Cereb Cortex 19:2810-2826.

Luo F, Wang Q, Kashani A, Yan J (2008) Corticofugal modulation of initial sound processing in the brain. J Neurosci 28:11615-11621.

Manis PB (1990) Membrane properties and discharge characteristics of guinea pig dorsal cochlear nucleus neurons studied in vitro. J Neurosci 10:2338-2351.

Matsubara JA, Phillips DP (1988) Intracortical connections and their physiological correlates in the primary auditory cortex (AI) of the cat. J Comp Neurol 268:38-48.

Merzenich MM, Brugge JF (1973) Representation of the cochlear partition of the superior temporal plane of the macaque monkey. Brain Res 50:275-296

Middlebrooks JC, Zook JM (1983) Intrinsic organization of the cat's medial geniculate body identified by projections to binaural response-specific bands in the primary auditory cortex. J Neurosci 3:203-224.

Middlebrooks JC, Dykes RW, Merzenich MM (1980) Binaural responsespecific bands in primary auditory cortex (AI) of the cat: topographical organization orthogonal to isofrequency contours. Brain Res 181:31-48.

Moeller CK, Kurt S, Happel MF, Schulze H (2010) Long-range effects of GABAergic inhibition in gerbil primary auditory cortex. Eur J Neurosci 31:49-59.

Montgomery N, Wehr M (2010) Auditory cortical neurons convey maximal stimulus-specific information at their best frequency. J Neurosci 30:13362-13366.

Oertel D, Bal R, Gardner SM, Smith PH, Joris PX (2000) Detection of synchrony in the activity of auditory nerve fibers by octopus cells of the mammalian cochlear nucleus. Proc Natl Acad Sci U S A 97:11773-11779.

Ojima H, Honda CN, Jones EG (1991) Patterns of axon collateralization of identified supragranular pyramidal neurons in the cat auditory cortex. Cereb Cortex 1:80-94.

Polley DB, Steinberg EE, Merzenich MM (2006) Perceptual learning directs auditory cortical map reorganization through top-down influences. J Neurosci 26:4970-4982.

Polley DB, Read HL, Storace DA, Merzenich MM (2007) Multiparametric auditory receptive field organization across five cortical fields in the albino rat. J Neurophysiol 97:3621-3638.

Popescu MV, Polley DB (2010) Monaural deprivation disrupts development of binaural selectivity in auditory midbrain and cortex. Neuron 65:718-731.

Portfors CV, Roberts PD, Jonson K (2009) Over-representation of speciesspecific vocalizations in the awake mouse inferior colliculus. Neuroscience 162:486-500.

Read HL, Winer JA, Schreiner CE (2001) Modular organization of intrinsic connections associated with spectral tuning in cat auditory cortex. Proc Natl Acad Sci U S A 98:8042-8047.

Read HL, Miller LM, Schreiner CE, Winer JA (2008) Two thalamic pathways to primary auditory cortex. Neuroscience 152:151-159.

Reyes AD, Rubel EW, Spain WJ (1994) Membrane properties underlying the firing of neurons in the avian cochlear nucleus. J Neurosci 14:5352-5364.

Richardson RJ, Blundon JA, Bayazitov IT, Zakharenko SS (2009) Connectivity patterns revealed by mapping of active inputs on dendrites of thalamorecipient neurons in the auditory cortex. J Neurosci 29:6406-6417.

Robertson D, Irvine DR (1989) Plasticity of frequency organization in auditory cortex of guinea pigs with partial unilateral deafness. J Comp Neurol 282:456-471.

Romanski LM, LeDoux JE (1993) Organization of rodent auditory cortex: anterograde transport of PHA-L from MGv to temporal neocortex. Cereb Cortex 3:499-514.

Rothschild G, Nelken I, Mizrahi A (2010) Functional organization and pop- 
ulation dynamics in the mouse primary auditory cortex. Nat Neurosci 13:353-360.

Sadagopan S, Wang X (2010) Contribution of inhibition to stimulus selectivity in primary auditory cortex of awake primates. J Neurosci 30:7314-7325.

Sanchez JT, Wang Y, Rubel EW, Barria A (2010) Development of glutamatergic synaptic transmission in binaural auditory neurons. J Neurophysiol 104:1774-1789.

Sanes DH, Geary WA, Wooten GF, Rubel EW (1987) Quantitative distribution of the glycine receptor in the auditory brain stem of the gerbil. J Neurosci 7:3793-3802.

Schreiner CE, Mendelson JR (1990) Functional topography of cat primary auditory cortex: distribution of integrated excitation. J Neurophysiol 64:1442-1459.

Schreiner CE, Winer JA (2007) Auditory cortex mapmaking: principles, projections, and plasticity. Neuron 56:356-365.

Smith DJ, Rubel EW (1979) Organization and development of brain stem auditory nuclei of the chicken: dendritic gradients in nucleus laminaris. J Comp Neurol 186:213-239.

Stiebler I, Neulist R, Fichtel I, Ehret G (1997) The auditory cortex of the house mouse: left-right differences, tonotopic organization and quantitative analysis of frequency representation. J Comp Physiol A 181:559-571.

Strumbos JG, Polley DB, Kaczmarek LK (2010) Specific and rapid effects of acoustic stimulation on the tonotopic distribution of $\mathrm{Kv} 3.1 \mathrm{~b}$ potassium channels in the adult rat. Neuroscience 167:567-572.

Tunturi AR (1962) Frequency arrangement in anterior ectosylvian auditory cortex of dog. Am J Physiol 203:185-193.

Wallace MN, Kitzes LM, Jones EG (1991) Intrinsic inter- and intralaminar connections and their relationship to the tonotopic map in cat primary auditory cortex. Exp Brain Res 86:527-544.

Wehr M, Zador AM (2003) Balanced inhibition underlies tuning and sharpens spike timing in auditory cortex. Nature 426:442-446.

Willott JF (1986) Effects of aging, hearing loss, and anatomical location on thresholds of inferior colliculus neurons in C57BL/6 and CBA mice. J Neurophysiol 56:391-408.
Willott JF, Aitkin LM, McFadden SL (1993) Plasticity of auditory cortex associated with sensorineural hearing loss in adult C57BL/6J mice. J Comp Neurol 329:402-411.

Winer JA, Sally SL, Larue DT, Kelly JB (1999) Origins of medial geniculate body projections to physiologically defined zones of rat primary auditory cortex. Hear Res 130:42-61.

Woolsey CN, Walzl EM (1942) Topical projection of nerve fibers from local regions of the cochlea to the cerebral cortex of the cat. Bull Johns Hopkins Hosp 71:315-344.

Wu GK, Li P, Tao HW, Zhang LI (2006) Nonmonotonic synaptic excitation and imbalanced inhibition underlying cortical intensity tuning. Neuron 52:705-715.

Wu GK, Arbuckle R, Liu BH, Tao HW, Zhang LI (2008) Lateral sharpening of cortical frequency tuning by approximately balanced inhibition. Neuron 58:132-143.

Xiao Z, Suga N (2002) Modulation of cochlear hair cells by the auditory cortex in the mustached bat. Nat Neurosci 5:57-63.

Young ED, Brownell WE (1976) Responses to tones and noise of single cells in dorsal cochlear nucleus of unanesthetized cats. J Neurophysiol 39:282-300.

Zhang LI, Tan AY, Schreiner CE, Merzenich MM (2003) Topography and synaptic shaping of direction selectivity in primary auditory cortex. Nature 424:201-205.

Zhang Y, Yan J (2008) Corticothalamic feedback for sound-specific plasticity of auditory thalamic neurons elicited by tones paired with basal forebrain stimulation. Cereb Cortex 18:1521-1528.

Zhang Y, Suga N, Yan J (1997) Corticofugal modulation of frequency processing in bat auditory system. Nature 387:900-903.

Zhang Y, Dyck RH, Hamilton SE, Nathanson NM, Yan J (2005) Disrupted tonotopy of the auditory cortex in mice lacking M1 muscarinic acetylcholine receptor. Hear Res 201:145-155.

Zhou Y, Liu BH, Wu GK, Kim YJ, Xiao Z, Tao HW, Zhang LI (2010) Preceding inhibition silences layer 6 neurons in auditory cortex. Neuron 65:706-717. 